Preprints of the

Max Planck Institute for

Research on Collective Goods

Bonn 2017/19

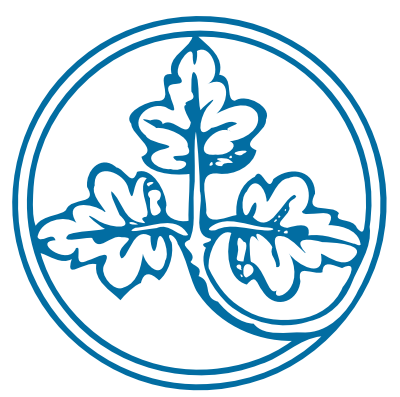

Finanzstabilität, Transparenz und Verantwortlichkeit:

Stellungnahme für das Bundesverfassungsgericht

Martin F. Hellwig

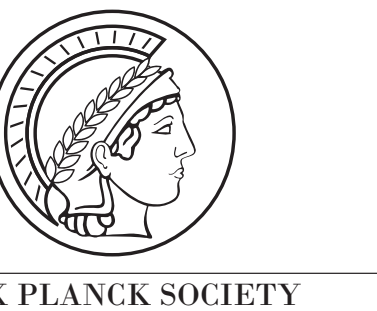




\section{Finanzstabilität, Transparenz und Verantwortlichkeit: Stellungnahme für das Bundesverfassungsgericht}

Martin F. Hellwig

September 2017 


\title{
Finanzstabilität, Transparenz und Verantwortlichkeit: Stellungnahme für das Bundesverfassungsgericht
}

\author{
Martin F. Hellwig
}

Zusammenfassung

Der Text beruht auf einer am 9. Mai 2017 vor dem Bundesverfassungsgericht abgegebenen Stellungnahme in der mündlichen Verhandlung zu einem Verfahren über den Umfang der Verpflichtung der Bundesregierung zur Information des Parlaments (Az. 2BvE 2/11). Das Gericht hatte um Auskünfte zur möglichen Gefährdung der Funktionsfähigkeit der Finanzmarktaufsicht und zur möglichen Gefährdung des Erfolgs der staatlichen Stützungsmaßnahmen durch eine öffentliche Beantwortung von Fragen der Bundestagsfraktion Bündnis 90/Die Grünen durch die Bundesregierung im November und Dezember 2010 gebeten.

Die Stellungnahme zeigt, dass die Argumente, mit denen die Bundesregierung eine öffentliche Beantwortung ablehnte, in großen Teilen fehlerhaft waren. Die Ablehnung half, eine öffentliche Diskussion über die Ursachen und die Verantwortung für die besondere deutsche Betroffenheit durch die Finanzkrise zu vermeiden. In Anbetracht der im internationalen Vergleich sehr hohen Kosten der Krise für den deutschen Steuerzahler wäre eine solche Diskussion angebracht gewesen, auch eine Diskussion darüber, wie zweckdienlich die seitherigen Reformmaßnahmen wirklich sein würden.

Die Stellungnahme vertritt die Auffassung, dass Einschränkungen des Anspruchs eines Finanzinstituts auf Vertraulichkeit vertretbar und ohne Schäden für die Funktionsfähigkeit der Aufsicht durchzusetzen sind, wenn diese Einschränkungen im Zusammenhang mit einer Staatshilfe stehen, die das Institut vor der Zahlungsunfähigkeit bewahrt hat. Bei Instituten, die eine Unterstützung durch den Steuerzahler benötigt und erhalten haben, war und ist das Risiko einer erneuten Destabilisierung durch Informationen über die Vergangenheit gering.

Die Stellungnahme weist auch die pauschale Vorstellung zurück, dass Transparenz an sich schon die Stabilität einzelner Banken oder gar des gesamten Finanzsystems gefährde, da jegliche Information panikartige Marktreaktionen hervorrufen könne. Modelle panikartiger Reaktionen auf „Sonnenflecken“, d.h. Informationen, die irrelevant sind oder keinen Neuigkeitswert haben, sind in der Wirtschaftstheorie populär, aber in der Realität werden Paniken durch neue Informationen ausgelöst, die die Erwartungen der Anleger über die wirtschaftlichen Aussichten substantiell beeinflussen. 


\section{Vorbemerkung}

Der folgende Text enthält die Ausarbeitung einer am 9. Mai 2017 in einer mündlichen Verhandlung vor dem Bundesverfassungsgericht abgegebenen Stellungnahme in einem Verfahren über die Verpflichtung der Bundesregierung zur Beantwortung parlamentarischer Anfragen (Az. 2BvE 2/11). Die Bundestagsfraktion Bündnis 90/Die Grünen hatte dagegen geklagt, dass die Bundesregierung die in verschiedenen Anfragen erbetene Information entweder verweigert hatte oder nur in der Geheimschutzstelle des Deutschen Bundestags gegeben hatte, so dass die Inhalte der öffentlichen Diskussion entzogen waren und auch die Parlamentarier, die in der Geheimschutzstelle Einsicht nahmen, eine solche Diskussion nicht initiieren konnten.

Ein Teil der Anfragen betraf die Arbeitsweise der Finanzaufsicht im Vorfeld der Finanzkrise von 2007-2009. Die Bundesregierung hatte die Verweigerung bzw. Geheimhaltung der erbetenen Auskünfte damit begründet, dass eine Offenlegung die Rechte der Betroffenen verletzen und die Funktionsfähigkeit der Finanzmarktaufsicht gefährden würde, ferner auch damit, dass bestimmte Informationen zu Marktreaktionen führen könnten, durch die die betroffenen Banken oder gar die Finanzstabilität insgesamt gefährdet und der Erfolg der staatlichen Stützungsmaßnahmen infrage gestellt würde. Das Gericht hatte mich um Auskünfte zur möglichen Gefährdung der Funktionsfähigkeit der Finanzmarktaufsicht und zur möglichen Gefährdung des Erfolgs der staatlichen Stützungsmaßnahmen durch eine öffentliche Beantwortung der zur Diskussion stehenden Anfragen gebeten. Die Inhalte der Anfragen und der Antworten der Bundesregierung werden im Anhang dieses Textes zusammengefasst wiedergegeben.

\section{Aufgabenstellung und Vorgehen}

Im Rahmen des Verfahrens über verschiedene Anfragen der Bundestagsfraktion Bündnis 90/Die Grünen zur Deutschen Bahn AG und zur Finanzmarktaufsicht (Az. 2BvE 2/11) hat das Bundesverfassungsgericht mich um Auskünfte zu Fragen der Gefährdung der Funktionsfähigkeit der Finanzmarktaufsicht und der Gefährdung des Erfolgs staatlicher Stützungsmaßnahmen durch diese Anfragen gebeten. Die vom Gericht gewünschten Auskünfte betreffen die Kleine Anfrage vom 11. November 2010 (BT-Drs. 17/3740) und die schriftlichen Anfragen vom 20. Dezember 2010 (Nr. 34 und Nr. 35, BTDrs. 17/4350) zur Arbeitsweise der Bundesanstalt für Finanzdienstleistungsaufsicht, zur IKB, und zum Umgang der Finanzmarktaufsicht mit Anleihenrückkäufen von Finanzinstituten.

Die Bundesregierung hat auf die genannten Anfragen die Antwort teils verweigert, teils nur in der Geheimschutzstelle des Deutschen Bundestags gegeben, so dass die Inhalte der öffentlichen Diskussion entzogen waren und auch die Parlamentarier, die in der Geheimschutzstelle Einsicht nahmen, eine solche Diskussion nicht initiieren konnten. Als Gründe wurden angegeben:

- die mangelnde Verfügbarkeit der verlangten Information,

- das Recht der beaufsichtigten Banken auf Diskretion der Aufsicht,

- das Recht der Vertragspartner auf Wahrung der vereinbarten Vertraulichkeit,

- die Furcht vor einer erneuten Gefährdung der betroffenen Banken,

- die Furcht vor einer Gefährdung des gesamten Finanzsystems.

Die vom Gericht gewünschten Auskünfte betreffen vor allem die beiden letztgenannten Gründe für die Zurückhaltung von Information. Meine Stellungnahme wird sich daher auf diese konzentrieren. Zur 
Einordnung der Überlegungen im Gesamtzusammenhang der Diskussion wird es aber sinnvoll sein, an der einen oder anderen Stelle auch auf die anderen Gründe einzugehen.

Die Kleine Anfrage vom 11. November hatte offensichtlich zum Ziel, die Arbeitsweise der Finanzmarktaufsicht im Vorfeld der Krise auf den Prüfstand zu stellen und die Ergebnisse ggfs. in die öffentliche Diskussion über die Finanzkrise und die erforderlichen Reformen einzubringen. Die von der Bundesregierung gegebenen Antworten waren geeignet, eine derartige Diskussion zu verhindern.

Das gilt z.B. für die Antwort zu Nr. 1 der Kleinen Anfrage vom 11. November 2010, betreffend die Häufigkeit von Wortmeldungen von Vertretern der Aufsicht und der Deutschen Bundesbank in Sitzungen von Aufsichtsräten und anderen Gremien der Banken: „Ein Wortprotokoll über diese Sitzungen wird regelmäßig nicht geführt. Wortmeldungen der Aufsicht sind damit für die Beantwortung der Anfrage nicht darstellbar.“ Für die Warnung vor „einem irreversiblen Vertrauensverlust ... mit entsprechender Reaktion des Markts“ ... und „erheblich negativen Auswirkungen auf die Stabilität des gesamten Finanzsystems“ als Folge einer Bekanntgabe der Zahl von Sonderprüfungen einzelner Institute in der Antwort zu Nr. 6 derselben Kleinen Anfrage gilt dasselbe.

Die zitierte Antwort zu Nr. 1 lässt die Frage offen, ob und inwiefern die Aufsicht im Vorfeld der Krise das ihr nach $\S 44$ Abs. 4 Satz 3 KWG zur Verfügung stehende Entsende- und Rederecht bei den ihrer Aufsicht unterstellten Instituten benutzt hat, um auf Risiken der von den Instituten verfolgten Strategien hinzuweisen. Für eine Behörde, die der Stabilität des Finanzsystems verpflichtet ist, ist der Umgang mit diesem Recht von grundsätzlicher Bedeutung, gerade auch wenn es um fundamentale Fehlentwicklungen geht. Man kann sich schwer vorstellen, dass die Erinnerung an die Wahrnehmung dieses Rechts in wichtigen Fällen am Fehlen von Wortprotokollen scheitert.

Vor diesem Hintergrund werde ich im folgenden ersten Hauptteil dieser Stellungnahme zunächst auf den Kontext der damaligen parlamentarischen Anfragen eingehen. Damit meine ich zum einen die Finanzkrise selbst, zum anderen das Ausmaß, in dem deutsche Institute und der deutsche Steuerzahler von der Finanzkrise betroffen waren, und zum dritten die politische Diskussion um die Krise und die nachherigen Reformen. Anschließend werde ich im zweiten Hauptteil diskutieren, was die verfügbaren wissenschaftlichen Erkenntnisse zum Zusammenhang von Transparenz und Finanzstabilität für die Diskussion um die Beantwortung der parlamentarischen Anfragen zur Finanzmarktaufsicht hergeben.

Meine Stellungnahme gründet sich nicht nur auf wissenschaftliche Untersuchungen, eigene und fremde, sondern auch auf Beobachtungen und Einsichten, die ich aufgrund verschiedener Beratungstätigkeiten gewonnen habe. $\mathrm{Zu}$ nennen sind insbesondere

- die Tätigkeit in dem von der Bundesregierung im Sommer 2010 bestellten Expertenrat zur Entwicklung von Strategien für den Ausstieg des Bundes aus den krisenbedingten Beteiligungen an Banken („Expertenrat Ausstiegsstrategien“),

- verschiedene Stellungnahmen für den Haushaltsausschuss und den Finanzausschuss des Bundestags, insbesondere zum Umgang mit Banken in Schieflagen,

- die Tätigkeit als Vorsitzender bzw. Stellvertretender Vorsitzender des Beratenden Wissenschaftlichen Ausschusses beim Europäischen Systemrisikoausschuss (2011-15). 


\section{Deutschland und die Finanzkrise(n)}

\subsection{Begrifflichkeit und Chronologie}

Die Bundesregierung macht geltend, „die Finanzkrise“ sei nicht schon 2009 beendet gewesen, sondern habe fortgedauert und auch 2010 noch eine große Verletzlichkeit von Finanzinstitutionen und Finanzsystem begründet. An dieser Aussage ist richtig, dass viele Fehlentwicklungen nicht korrigiert wurden, teilweise bis heute nicht. Jedoch ist der Ausdruck „die Finanzkrise“ als Bezeichnung für die Entwicklung der letzten zehn Jahre ungenau und irreführend. Aus deutscher Sicht sind verschiedene Krisen zu unterscheiden:

- die von den Immobilienfinanzierungen und Hypothekenverbriefungen in den USA ausgehende Krise des internationalen Finanzsystems in den Jahren 2007-2009,

- die irische Immobilien- und Bankenkrise (2008-2010),

- die spanische Immobilien- und Bankenkrise (2010-2012),

- die südeuropäischen Staatsschuldenkrisen (ab 2010),

- die Krise der Seeschifffahrt (ab 2008) und der auf Schiffsfinanzierungen spezialisierten Banken (ab 2013),

- die Turbulenzen des europäischen Finanzsystems im zweiten Halbjahr 2011,

- die griechischen und italienischen Krisen der Unternehmenskredite.

Diese Krisen sind insofern Teile eines Gesamtzusammenhangs als sie alle auf übermäßige Kreditvergabe in den Jahren vor 2008 zurückgehen. Jedoch fanden die Krisen nicht alle gleichzeitig statt. Auch waren die verschiedenen Krisen nicht für alle Institute und alle Länder in gleicher Weise von Bedeutung. So waren etwa US-amerikanische Banken kaum von den verschiedenen europäischen Krisen betroffen. Und deutsche Banken wurden von den Auswirkungen der irischen und spanischen Immobilienkrisen dadurch verschont, dass in diesen Ländern jeweils der Staat die Inhaber vorrangiger ungesicherter Schuldtitel der heimischen Banken schadlos hielt.

In den Anhörungen des Expertenrats Ausstiegsstrategien im zweiten Halbjahr 2010 war nichts von der Gefahr eines erneuten Ausbruchs der Finanzkrise zu hören. Die Verantwortlichen der betroffenen Banken gingen von einem Fortgang der Stabilisierung des Systems aus. Auch der Bericht des Expertenrats beruhte auf dieser Hypothese, so etwa die in diesem Bericht enthaltenen Vorschläge zum Umgang mit der staatlichen Beteiligung an der Commerzbank. ${ }^{1}$

Diese Vorschläge zielten darauf ab, dass die Bank die Stille Einlage des Bundes in Höhe von € 16,4 Mrd. weitgehend zurückzahlen sollte. Für diese Stille Einlage bestand eine Verpflichtung zur Zahlung eines Couponzinses von 9 \% in jedem Jahr, in dem die Bank nach HGB-Rechnungslegung einen Gewinn auswies. Diese Verpflichtung stand einer nachhaltigen Rekapitalisierung durch Gewinnthesaurierung entgegen, machte es der Bank auch praktisch unmöglich, neues Eigenkapital am Markt aufzunehmen. Da die Stille Einlage des Bundes aufsichtsrechtlich als Eigenkapital behandelt wurde, bedeutete die Rückzahlung eine Reduktion des aufsichtsrechtlichen Eigenkapitals; die Vorschläge des Expertenrats gingen dabei bis an die Grenze dessen, was aufsichtsrechtlich möglich war. Dies wäre nicht geschehen, wenn man zu diesem Zeitpunkt mit wesentlichen neuen Risiken gerechnet hätte.

1 „Strategien für den Ausstieg des Bundes aus krisenbedingten Beteiligungen an Banken: Gutachten des von der Bundesregierung eingesetzten Expertenrats“, 24. Januar 2011,

http://www.bundesfinanzministerium.de/Content/DE/Standardartikel/Themen/Internationales_Finanzmar kt/Finanzmarktpolitik/2011-02-15-gutachten-bankenbeteiligung-anlage.pdf;

jsessionid=35E06CB0ECE36D5AB3DD7120BF512D9E?_blob=publicationFile\&v=3, abgerufen am 8. Mai 2017. 
Die Commerzbank, an der der Bund zu diesem Zeitpunkt nicht nur mit der Stillen Einlage, sondern auch mit $25 \%$ des Aktienkapitals (plus einer Aktie), beteiligt war, verwirklichte im ersten Halbjahr 2011 die Vorschläge des Expertenrats bzw. eine noch weitergehende Variante und zahlte von der Stillen Einlage $€ 14$ Mrd. zurück. Diese Maßnahme brachte das Eigenkapital der Bank an die Grenze des von der Aufsicht Geforderten und hatte zur Folge, dass die Bank in den Turbulenzen des zweiten Halbjahrs 2011 und im Umgang mit der vom EU-Gipfel im Oktober 2011 verordneten Erhöhung der Eigenkapitalanforderungen in erhebliche Bedrängnis kam. Hätte man die spätere Gefährdung vorhergesehen, so wäre man vermutlich anders vorgegangen, von Seiten der Bank und von Seiten des Bundes, der an den Maßnahmen des ersten Halbjahrs 2011 beteiligt war. Als Mitglied des Expertenrats hätte ich selbst jedenfalls vor dem gewählten Vorgehen gewarnt.

Die Turbulenzen des europäischen Finanzsystems im zweiten Halbjahr 2011 wurden durch die Wende der Politik in der Frage einer Verlustbeteiligung der privaten Gläubiger in der griechischen Staatsschuldenkrise verursacht. Beim deutsch-französischen Treffen im Oktober 2010 in Deauville hatte es geheißen, in Zukunft sollten bei Zahlungsunfähigkeit von Staaten die privaten Gläubiger an den Verlusten beteiligt werden, das aber erst ab 2013. Im November 2011 verkündeten die Finanzminister der Eurozone, diese Verlustbeteiligung privater Gläubiger werde es nur bei Insolvenz, nicht aber bei Illiquidität des betreffenden Staats geben. Im März 2011 beschlossen die Finanzminister der Eurozone, es solle alsbald auf die ausstehenden Staatsschulden Griechenlands einen Schuldenschnitt geben, d.h. eine Verlustbeteiligung der privaten Gläubiger. Im Juli 2011 setzten die Finanzminister für den Schuldenschnitt einen Prozentsatz von 21 \% fest. Im Laufe des Monats August wurde deutlich, dass das nicht ausreichen würde und der Schuldenschnitt über 50 \% liegen würde.

Parallel zu dieser Entwicklung zogen US-amerikanische Geldmarktfonds ihre Mittel von europäischen Banken ab - und zwar vor allem von denen, deren Solvenz durch den Schuldenschnitt besonders gefährdet sein würde, so etwa der belgisch-französischen Bank Dexia. Der Abzug dieser Mittel veranlasste die europäischen Banken ihrerseits, Vermögenswerte zu verkaufen, um ihre Liquidität zu erhalten. Die dadurch verursachte Baisse der Wertpapierbörsen verstärkte die Zweifel der Investoren an der Solvenz dieser Banken. Die vom EU-Gipfel im Oktober 2011 verordnete Erhöhung der Eigenkapitalanforderungen sollte diesen Zweifeln entgegenwirken, hatte aber zunächst nur zur Folge, dass Banken noch weiter Vermögenswerte verkauften, nunmehr mit dem Ziel, ihre Bilanzen zu verkürzen, so dass sie die erhöhten Eigenkapitalanforderungen erfüllen könnten, ohne zusätzliche eigene Mittel aufzunehmen.

Im Juni 2011 nahm ich als Vorsitzender des Beratenden Wissenschaftlichen Ausschusses beim Europäischen Systemrisikoausschuss an der Sitzung des Governing Board dieser Institution teil. Im Zentrum der Diskussion von Systemrisiken in dieser Sitzung stand der Stress Test der Europäischen Bankenaufsicht (EBA), dessen Ergebnisse Mitte Juli veröffentlicht werden sollten. Die Hauptsorge betraf die Frage, ob alle Aufsichtsbehörden und alle Mitgliedstaaten hinreichend auf die Möglichkeit vorbereitet seien, dass der Stress Test bei der einen oder anderen Bank Korrekturmaßnahmen erforderlich machen könnte, z.B. durch Zufuhr zusätzlichen Eigenkapitals. Der im vorstehenden Abschnitt beschriebene Zusammenhang von Schuldenschnitt, Bedrohung der Solvenz, Mittelabzug der Gläubiger und Kurssenkungen in den Wertpapiermärkten wurde nicht thematisiert. Allerdings wurde die Bedeutung dieses Zusammenhangs erst in den Folgemonaten deutlich, zum einen, weil die Erwartungen über die Höhe des Schuldenschnitts wie oben beschrieben deutlich pessimistischer wurden, zum anderen, weil die Europäische Bankenaufsicht gleichzeitig mit den Ergebnissen des Stress Tests auch die Bestände der einzelnen Banken an griechischen Staatsschulden veröffentlichte, so dass die Implikationen des Schuldenschnitts für die Solvenz der Institute deutlich wurden. 
Gewiss hat die sogenannte „Euro-Krise“ schon im Frühjahr 2010 begonnen. Hätten die europäischen Institutionen zu diesem Zeitpunkt eine Intervention abgelehnt, entsprechend dem Wortlaut der Verträge, so hätten die privaten Gläubiger und Griechenland unmittelbar über eine Umschuldung bzw. einen Schuldenschnitt verhandeln müssen. Zu diesem Zeitpunkt hielten deutsche Banken griechische Staatsschulden in Höhe von über $€ 20$ Mrd. (französische Banken über $€ 30$ Mrd.), und ein Schuldenschnitt hätte unmittelbar die Solvenz gefährdet. Die Intervention der europäischen Institutionen, Gründung des EFSF und später des ESM, nahm den privaten Gläubigern dieses Risiko zunächst ab, so dass die „Euro-Krise“ im weiteren Verlauf nicht mehr eine Krise des europaweiten Finanzsystems war, sondern eine Krise der betroffenen Staaten und der europäischen Institutionen - bis 2011 der Schuldenschnitt beschlossen und konkretisiert wurde.

Etliche deutsche (und französische) Banken scheinen diese Phase genutzt zu haben, um griechischen Staatsanleihen zu verkaufen, vermutlich an griechische und zypriotische Banken, die dann durch den Schuldenschnitt 2012 insolvent wurden und Staatshilfen zur Rekapitalisierung benötigten. ${ }^{2}$ Im Vergleich zum März 2010 sind die im Zusammenhang mit dem EBA-Stress Test von 2011 veröffentlichten Griechenland-Positionen deutscher Banken jedenfalls deutlich niedriger.

Für das zweite Halbjahr 2010 ist ansonsten noch die irische Bankenkrise zu nennen. Auf dem Höhepunkt der Lehman-Krise 2008 hatte die Republik Irland eine Staatsgarantie für die Schuldtitel irischer Banken ausgesprochen, um die Refinanzierung dieser Banken sicherzustellen. Im Herbst 2010 lief diese Garantie aus; gleichzeitig war deutlich geworden, dass aufgrund der irischen Immobilienkrise viele der von irischen Banken vergebenen Kredite notleidend und die Banken selbst insolvent waren. Bei der Bereinigung der Krise stand zur Diskussion, unter Ausnutzen des Auslaufens der Garantie die Gläubiger der Banken entsprechend dem geltenden Insolvenzrecht an den Verlusten der Banken zu beteiligen, nicht nur die nachrangigen, sondern auch die ungesicherten vorrangigen Gläubiger. Ein solches Vorgehen hätte deutsche Banken erheblich gefährdet. Die irische Regierung nahm jedoch davon Abstand, da die Europäische Zentralbank (EZB) für diesen Fall mit einer Untersagung der Notkredite für irische Banken gedroht hatte. ${ }^{3}$ Auch in diesem Fall hat also die Intervention der europäischen Institutionen dafür gesorgt, dass private Gläubiger, hier vor allem deutsche Geschäftsbanken, vor den Konsequenzen fehlerhafter Geschäftsentscheidungen bewahrt wurden. Auch dieses Risiko für die Finanzstabilität entfiel.

Aus den vorstehend skizzierten Entwicklungen ziehe ich folgende Schlüsse:

- Neue Information kann Finanzturbulenzen auslösen. Das gilt allerdings nicht für jede Information; z. B. die übrigen mit den Ergebnissen des Stress Tests von 2011 veröffentlichten Informationen hatten keine erkennbaren Wirkungen. Finanzturbulenzen sind vielmehr dann zu erwarten, wenn die Informationen, um die es geht, die Einschätzungen der Marktteilnehmer über zukünftige wirtschaftliche Entwicklungen, in diesem Fall die Belastungen der Banken durch den griechischen Schuldenschnitt, maßgeblich beeinflussen.

2 Das Griechenland-Portefeuille von Hypo Real Estate (HRE) ging im Herbst 2010 zum Nennwert, d.h. ohne Abschlag, an FMS Wertmanagement, die vom Bund für die zweifelhaften Vermögenswerte von HRE eingerichtete „Bad Bank“. Die Europäische Kommission schätzte die mit der Übertragung verbundene Beihilfe auf $€ 16$ Mrd. Siehe S.M. Cas und I. Peresa (2016), What makes for a Good 'Bad Bank'? The Irish, Spanish and German Experience, Discussion Paper 036/September 2016, Directorate General for Economic and Financial Affairs, European Commission, http://ec.europa.eu/info/publications/whatmakes-good-bad-bank-irish-spanish-and-german-experience_en .

3 Brief des Präsidenten der EZB an den irischen Premierminister vom 19.11.2010, http://www.ecb.europa.eu/press/html/irish-letters.en.html, abgerufen am 3. Juni 2017. 
- Die Turbulenzen des europäischen Finanzsystems im zweiten Halbjahr 2011 sind nicht so sehr ein Ergebnis einer seit 2007 andauernden Gefährdung des Finanzsystems als vielmehr ein Ergebnis einer Richtungsänderung der Politik in Sachen Verlustbeteiligung der privaten Inhaber von griechischen Schuldtiteln, durch die die Solvenz der betroffenen Institute unmittelbar gefährdet wurde.

- Im zweiten Halbjahr 2010 bestand weitgehend die Auffassung, die Gefahr einer Krise sei deutlich zurückgegangen. Die Zustimmung der Bundesregierung zu den im ersten Halbjahr 2011 vorgenommenen Kapitalmaßnahmen der Commerzbank lässt vermuten, dass auch die Bundesregierung diese Auffassung teilte.

- Bei der Analyse von Krisen und Turbulenzen ist es wichtig, die Wirkungsmechanismen im Detail zu untersuchen, im vorstehend diskutierten Fall der Krise des zweiten Halbjahrs 2011 das Zusammenspiel von Solvenzproblemen, Abhängigkeit von Geldmarktfonds und Wertpapiermärkten.

\subsection{Warum gab es keine Untersuchung der deutschen Betroffenheit durch die Krise(n)?}

Für die deutschen Steuerzahler waren die Fehlentwicklungen des Finanzsystems seit 2000 sehr teuer. Für die Endabrechnung erwarte ich, dass die Kosten für die Steuerzahler insgesamt auf deutlich mehr als $€ 70$ Mrd. kommen werden. Eine genaue Aufstellung ist noch nicht möglich, da viele Zahlen nicht bekannt sind und im Übrigen viele Vorgänge noch nicht abgeschlossen sind. Folgende Zahlen sind öffentlich genannt worden: WestLB $€ 18$ Mrd. (laut NRW-Finanzminister Walter-Borjans), ${ }^{4}$ Industriekreditbank € 9,6 Mrd., ${ }^{5}$ Hypo Real Estate ca. € 14 Mrd. ${ }^{6}$, HSH Nordbank $€ 16$ Mrd. (laut Ministerpräsident Albig) ${ }^{7}$, Sächsische Landesbank mindestens $€ 1,4$ Mrd., ${ }^{8}$ Landesbank Baden-Württemberg $€$ 5 Mrd., ${ }^{9}$ Bayern LB $€ 10$ Mrd., ${ }^{10}$ Commerzbank $€ 3-6$ Mrd. $^{11}$

4 Siehe http://www.handelsblatt.com/finanzen/banken-versicherungen/abwicklung-westlb-kostetsteuerzahler-18-milliarden-euro/6775470.html abgerufen am 8. Mai 2017.

5 Siehe C. Kaserer, Staatliche Hilfen von Banken und ihre Kosten, Initiative Neue Soziale Marktwirtschaft: Texte zur Sozialen Marktwirtschaft 2, und Technische Universität München, August 2010.

6 Siehe die Rechnungen von A. Storn, Das Desaster der HRE-Rettung: 237 Euro von jedem, Die Zeit, 26. September 2013, http://www.zeit.de/2013/40/rettung-hypo-real-estate/seite-2 und A. Storn, Zur Sonne, zur Freiheit, Die Zeit, 30. Juli 2015, http://www.zeit.de/2015/29/welt-des-geldes-frankfurt. Die hohen Kosten der HRE-Rettung erklären sich zu guten Teilen aus Verlusten aufgrund des griechischen Schuldenschnitts. Siehe S.M. Cas und I. Peresa a.a.O. (Fn. 2).

7 Siehe http://www.zeit.de/hamburg/politik-wirtschaft/2017-01/steuergelder-hsh-nordbank-hamburgschleswig-holstein.

8 Dieser Betrag wurde bis 2016 von dem vom Land Sachsen eingerichteten Garantiefonds abgerufen; es ist nicht auszuschließen, dass der Garantiefonds am Ende in voller Höhe (€ 2,75 Mrd.) in Anspruch genommen werden wird.

$9 \quad$ Kaserer a.a.O. (Fn. 5).

10 Kaserer a.a.O. (Fn. 5). Die Zahlen für LBBW und Bayern LB entsprechen den von öffentlichen Trägern eingebrachten Beträgen zur Rekapitalisierung der Banken. Diese Zahlen entsprechen näherungsweise auch den in den Krisenjahren ausgewiesenen Verlusten dieser Banken.

11 Hier handelt es sich um die Wertverluste des Bundes auf Aktien der Commerzbank. Der Bund hatte nicht nur anfangs $€ 1,8$ Mrd. für $25 \%$ des Aktienkapitals bezahlt, sondern auch später, als die Bank neues Eigenkapital aufnahm, um die Stille Einlage zurückzuzahlen, sein Aktienengagement erhöht. 
Diese Kosten der Bankenrettungen sind auch im internationalen Vergleich sehr hoch. Kosten in ähnlicher Höhe oder darüber, zumindest in Relation zum Bruttosozialprodukt, finden sich sonst bisher nur in Ländern wie Island, Irland und Spanien, die selbst im Zentrum von Bankenkrisen standen, sowie Griechenland, wo die Banken vom staatlichen Schuldenschnitt betroffen waren. In den USA und der Schweiz hat der Staat mit seinen Interventionen zur Stützung von Banken sogar Gewinne erzielt.

In den USA richtete der Kongress 2009 eine unabhängige Kommission zur Untersuchung der Finanzkrise ein, die Financial Crisis Inquiry Commission. Der Bericht dieser Kommission behandelt sehr ausführlich die Entwicklungen des Finanzsektors und der Finanzregulierung vor der Krise, den Aufbau der Immobilienblase und der Risiken, das Zerplatzen der Blase und die verschiedenen Ansteckungsmechanismen. ${ }^{12}$ Der Bericht geht auch jeweils im Detail auf einzelne Institute ein. Er wurde im Januar 2011 veröffentlicht. Von einer Auswirkung der Veröffentlichung auf die betroffenen Institutionen oder auf das Finanzsystem insgesamt ist mir nichts bekannt.

In Deutschland hat es keine vergleichbare Untersuchung gegeben. Es gab 2009 einen Untersuchungsausschuss des Bundestags zu Hypo Real Estate, aber der Bericht dieses Untersuchungsausschusses ist weit von dem entfernt, was die Financial Crisis Inquiry Commission geliefert hat. Der Bericht des Untersuchungsausschusses bezeichnet die Krise ohne eigentliche Untersuchung pauschal als Ergebnis von Fehlentwicklungen in den USA, denen Deutschland zum Opfer fiel, zunächst der Fehlentwicklungen bei der Hypothekenverbriefung, sodann der allgemeinen Panik nach dem Lehman-Konkurs.

Die Frage, warum Deutschland von diesen Fehlentwicklungen so viel stärker betroffen war als etwa Frankreich, Italien oder Spanien wird nicht gestellt. Soweit die Entwicklungen in Deutschland thematisiert werden, enthält der Bericht lange Texte mit wörtlichen Zitaten der Berichte handelnder Personen ohne nennenswerte Analyse. Das Handeln der Personen wird im Hinblick auf die Frage untersucht, ob geltende Vorschriften verletzt wurden oder nicht; die Frage, ob die Vorschriften angemessen waren bzw. ob die Verantwortlichen eine Unangemessenheit von Vorschriften frühzeitig hätten erkennen können oder müssen, wird nicht behandelt.

\subsection{Zu den Ursachen der besonderen deutschen Betroffenheit durch die Krisen}

Eine auf die Ursachen der besonderen deutschen Betroffenheit durch die Krise gerichtete Untersuchung hätte sich folgende Fragen vornehmen können:

- Welche Rolle spielte die mit dem Pfandbriefgesetz von 2005 verbundene Erleichterung des Zugangs zu diesem Segment des Finanzsystems?

- Warum waren die Landesbanken so stark von der Krise betroffen?

- Warum hat man die Problematik der Verbriefungen und der Zweckgesellschaften nicht rechtzeitig erkannt oder erkennen wollen?

- Warum ist die Abhängigkeit der Banken von Geldmarktfinanzierungen in den vergangenen zwei Jahrzehnten so stark angestiegen und welche Risiken sind damit verbunden? 
Im Folgenden erläutere ich diese Fragen etwas näher.

Pfandbriefbanken: Zu den Fehlentwicklungen der Jahre vor der Finanzkrise gehört es, dass ein Institut wie Hypo Real Estate (und ganz ähnlich die belgisch-französische Bank Dexia) extreme Fristentransformation betrieb, d.h. für die Finanzierung der bei der Emission eines Pfandbriefs erforderlichen Überdeckung, d.h. des Betrags, um den die als Sicherheiten für den Pfandbrief dienenden Wertpapiere den Nennwert des Pfandbriefs überstiegen, extrem kurzfristige Schulden auf dem Geldmarkt aufnahm. Ein Grund für diese Fehlentwicklung lag darin, dass der Wettbewerb in diesem Marktsegment sehr intensiv war, die Margen daher sehr gering waren und man extreme Fristentransformation betreiben musste, um überhaupt im Markt überleben zu können. Für Institute wie Eurohypo oder Westimmo als Töchter von Commerzbank und WestLB war das weniger problematisch, denn diese Banken finanzierten sich selbst auch durch kurzfristige Einlagen, von Endkunden oder Sparkassen. Hypo Real Estate konnte das nicht, war daher auf den Geldmarkt angewiesen und unmittelbar vom Zusammenbruch der Geldmärkte im September 2008 betroffen. ${ }^{13}$ Im Bericht des Untersuchungsausschusses wird die Problematik dieses Finanzierungsmodells verschiedentlich angesprochen, die Gründe dafür und ein etwaiger Reformbedarf aber ausgeklammert.

Landesbanken: Warum hatten die Landesbanken so stark in Hypothekenverbriefungen in den USA investiert? Welche Rolle spielte es, dass Landesbanken nach der Einigung der Bundesregierung mit der Europäischen Kommission zum Auslaufen der staatlichen Gewährträgerhaftung in der Übergangszeit von 2001 bis 2005 noch einmal in sehr großem Umfang Schulden mit Ländergarantien aufgenommen hatten und die Erlöse nun anlegen mussten? Inwiefern beruhte das desaströse Engagement der Bayern LB bei der österreichischen Hypo Group Alpe-Adria auf Nachlässigkeit bei der due diligence? Welche Rolle spielte es, dass die den Landesbanken zugänglichen Geschäftsbereiche von jeher durch intensiven Wettbewerb charakterisiert sind und die Wettbewerbsintensität sich in den 1990er Jahren noch einmal erhöht hatte? ${ }^{14}$

Verbriefungen und Zweckgesellschaften: Dass die vollständige Übertragung von Kreditrisiken im Zuge der Verbriefung negative Anreizwirkungen auf die Kreditwürdigkeitsprüfung hat, sagt schon der gesunde Menschenverstand. ${ }^{15}$ Und ebenso offensichtlich musste es sein, dass die Möglichkeit der Auslagerung großer Bestände an Verbriefungen in Zweckgesellschaften ohne eigene Mittel die für diese Auslagerung verantwortlichen Banken erheblichen Liquiditäts- und Solvenzrisiken aussetzte, denn die Zweckgesellschaften unterlagen nicht den normalen aufsichtsrechtlichen Regeln. ${ }^{16}$ Die Verluste bei IKB, Sachsen LB und WestLB wären ohne diese Möglichkeiten kaum so groß geworden wie sie es wurden. Maßgebliche Vertreter des Bundesministeriums der Finanzen hatten die Entwicklung von Verbriefungen und von Zweckgesellschaften begrüßt und gefördert. ${ }^{17}$ Es wäre wichtig, zu wissen, ob

13 Siehe Expertenrat Ausstiegsstrategien, a.a.O. (Fn. 1), Kap. 3, sowie M. Hellwig, Stellungnahme für den Haushaltsausschuss des Deutschen Bundestags zum Entwurf eines Dritten Gesetzes zur Umsetzung eines Maßnahmenpakets zur Stabilisierung des Finanzmarktes (3. FMStG), 7. November 2012.

14 Siehe Expertenrat Ausstiegsstrategien, a.a.O. (Fn. 1), Kap. 3, sowie M. Hellwig (2010), a.a.O. (Fn. 13).

15 Zur Einordnung dieses Problems siehe M. Hellwig (1998), Banks, Markets, and the Allocation of Risks in an Economy, Journal of Institutional and Theoretical Economics 154, 328 - 348, insbes. 334 - 337, ferner M. Hellwig (2009), Systemic Risk in the Financial Sector: An Analysis of the Subprime-Mortgage Financial Crisis, De Economist 157, 129-207.

16 Siehe M. Hellwig (2009), a.a.O. (Fn. 15), ferner A. Admati und M. Hellwig, Des Bankers neue Kleider, Finanzbuchverlag München, Kap. 13.

17 J. Asmussen, Verbriefungen aus Sicht des Bundesfinanzministeriums, Kredit und Kapital 19/2006, 10 12. M. Thiemann, Out of the shadow? Accounting for Special Purpose Entities in European banking systems, Competition and Change 16 (2012), 37-55, zeigt, dass Toleranz gegenüber der Verwendung von Zweckgesellschaften zur Umgehung der international vereinbarten Vorschriften zur Bankenregulierung 
und inwiefern dieses anfängliche Engagement einen Einfluss darauf hatte, dass die mit Verbriefungen und Zweckgesellschaften verbundenen Fehlentwicklungen in Deutschland erst korrigiert wurden, als es zu spät war. Die Antwort der Bundesregierung auf die diesbezügliche Frage Nr. 8 der Kleinen Anfrage der Fraktion Bündnis 90/Die Grünen vom 11. November 2010 gibt darüber keinen Aufschluss. Das in der Antwort auf Frage Nr. 1 dieser Kleinen Anfrage behauptete Fehlen von Unterlagen zu Interventionen der Aufsicht in Gremiensitzungen von Banken gibt zu der Vermutung Anlass, dass man diese Fehlentwicklung tatsächlich weitgehend ignorierte und erst im Nachhinein ein Unbehagen geltend macht.

Abhängigkeit vom Geldmarkt: Der Bericht des HRE-Untersuchungsausschusses nimmt diese Abhängigkeit als gegeben an und sieht die deutschen Banken als Opfer des Zusammenbruchs des Geldmarkts in der Panik nach dem Konkurs von Lehman Brothers. Bei näherer Betrachtung stellt man allerdings fest, dass (i) die Abhängigkeit großer Banken von bestimmten Geldmarktrefinanzierungen neueren Datums ist, dass (ii) der Zusammenbruch dieser Märkte nach dem Lehman-Konkurs nicht als Ergebnis einer allgemeinen Panik anzusehen ist, sondern als Ergebnis bestimmter Wirkungszusammenhänge, die später auch die Turbulenzen von 2011 prägten und grundsätzlich auch heute noch systemische Risiken begründen.

Der Anstieg der Bedeutung des Geldmarktes für die Finanzierung von Banken in den Jahren seit 1995 spiegelt drei Entwicklungen: Zum einen die zunehmende Bedeutung von Geldmarktfonds und anderen Schattenbanken in den USA und ein Strategiewechsel von vielen dieser Geldmarktfonds hin zur Finanzierung von Banken und Zweckgesellschaften von Banken und weg vom Ankauf kurzfristiger Schuldtitel des Staates und der Unternehmen außerhalb des Finanzsektors; dieser Strategiewechsel wurde teilweise verursacht durch die zunehmende Konkurrenz Chinas als Käufer solcher Titel. Zum anderen das starke, durch zunehmende Verschuldung finanzierte Wachstum der großen Banken; ${ }^{18}$ dieses wurde ermöglicht durch den Einsatz bankinterner Modelle zur Bestimmung des aufsichtsrechtlich erforderlichen Eigenkapitals und durch den Einsatz von Zweckgesellschaften, die nicht den aufsichtsrechtlichen Vorschriften unterworfen waren. ${ }^{19}$ Zum Dritten schließlich Änderungen der in den USA geltenden rechtlichen Regeln für mit Sicherheiten unterlegte kurzfristige Schuldtitel wie asset-backed commercial paper und Repo-Kredite; diese Änderungen betrafen zum einen die regulatorische Behandlung der Garantien von Banken für Zweckgesellschaften, die diese Titel ausgaben, zum anderen den Umgang mit den Sicherheiten in einem etwaigen Konkursverfahren. ${ }^{20}$ Die mit diesen Instrumenten verbundenen Systemischen Risiken waren nicht bedacht worden.

Lehman Brothers musste in den Konkurs gehen, als die Gläubiger nicht mehr an die Werthaltigkeit der Sicherheiten glaubten und die erforderlichen Anschlussfinanzierungen verweigerten. Infolge des

in den Ländern besonders ausgeprägt war, in denen die Regierungen „ihre“ Banken als „Champions“ betrachteten und im internationalen Wettbewerb zu fördern versuchten.

18 Siehe Advisory Scientific Committee of the European Systemic Risk Board, Is Europe Overbanked? Report 04/2014.

19 Der Einsatz bankinterner Modelle wird zumeist mit dem 2004 verabschiedeten, unter dem Namen „Basel II“ bekannten internationalen Regelwerk verknüpft. Für die Entwicklungen, die zur Krise führten, waren allerdings schon die Regeln verantwortlich, die 1996 als Ergänzung des ersten Basler Abkommens für die Eigenkapitalunterlegung von Anlagen im Handelsbuch („Marktrisiken“) verabschiedet worden waren. Bei großen europäischen Banken und den US-amerikanischen Investment-Banken machte die Eigenkapitalunterlegung dieser Anlagen weniger als 1\% aus. Siehe Financial Services Authority, The Prudential Regime for Trading Activities: A Fundamental Review, Discussion Paper 4/2010, London.

$20 \mathrm{Zu}$ asset-backed commercial paper und zu Zweckgesellschaften siehe V. Acharya, P. Schnabl und G. Suarez (2013), Securitization without Risk Transfer, Journal of Financial Economics 107, 515-536. Zu Repo-Krediten, siehe Admati und Hellwig, a.a.O. (Fn. 15), Kap. 10. 
Lehman-Konkurses musste der US-amerikanische Geldmarktfonds Reserve Primary Verluste verbuchen, dadurch sank der Wert eines Fonds-Anteils von 1 US \$ auf 0,97 US \$. Das Absinken des Anteilswerts unter 1 US \$ löste einen Sturm der Anteilsinhaber aus; in fünf Tagen wurden Anteile im Wert von US \$ 60 Mrd. von Reserve Primary abgezogen, von einem Anfangsbestand von US \$ 62 Mrd. ${ }^{21}$ Auch andere Geldmarktfonds erlebten drastische Mittelabzüge, so dass die US-Regierung sich am Ende der Woche veranlasst sah, eine Art Einlagenversicherung für Geldmarktfonds einzuführen, um die Mittelabzüge zu stoppen. Dass europäische Banken wie Hypo Real Estate Ende September 2008 keine Geldmarktkredite mehr bekamen, lag nicht so sehr daran, dass diesen Banken nicht mehr getraut wurde, als vielmehr daran, dass viele Geldmarktfonds als Geldgeber ausfielen, weil ihnen selbst die Geldgeber abhandengekommen waren. ${ }^{22}$ Die Reaktion der Banken, zur Sicherstellung der eigenen Liquidität Wertpapiere zu verkaufen, ließ allerdings die Wertpapierkurse weltweit drastisch sinken, was wiederum zu Fragen nach der Solvenz der Banken Anlass gab.

Ähnliche Mechanismen spielten, wie erwähnt, auch in den Turbulenzen von 2011 eine wichtige Rolle. Die hier beschriebene Verletzlichkeit des Finanzsystems von den Geldmarktfonds und - indirekt - den Anteilseignern der Geldmarktfonds ist nach wie vor gegeben. Eine Analyse der Zusammenhänge, die über die pauschale Berufung auf die Panik nach dem Lehman-Konkurs hinausginge, hätte die Möglichkeit geboten, über die aufsichtsrechtlichen Rahmenbedingungen zu diskutieren, die diese krisenträchtige Entwicklung des Finanzsystems ermöglichten.

\subsection{Zum Umgang mit Banken in Schieflagen: Bankenrestrukturierungsgesetz und Ausstiegsstrategien}

Zu den Absurditäten des deutschen Umgangs mit der Finanzkrise gehört es, dass die Bundesregierung zuerst erhebliche Mittel aufwandte, um die Bank Hypo Real Estate vor der Zahlungsunfähigkeit zu bewahren, und hinterher noch $€ 1,30$ pro Aktie bezahlen musste, um die vollständige Kontrolle über die Bank zu bekommen. Ohne die vorherige Stützungsaktion wäre die Bank zahlungsunfähig geworden, die Aktionäre hätten die Kontrolle verloren, und die Aktien wären nichts mehr wert gewesen.

Die Absurdität erklärt sich dadurch, dass die Stützungsmaßnahmen vom Herbst 2008 durchgeführt wurden, ohne dass der Bund, der das Geld dafür bereitstellte, sich entsprechende Kontrollrechte sicherte, anders als etwa die schwedische Regierung in der Krise von 1992. Die Zurückhaltung des Bundes hatte politische und rechtliche Gründe. Zum einen wollte man dem Vorwurf vorbeugen, man nutze die Krise, um das Banksystem zu verstaatlichen (bzw. die Landesbanken zu verbundlichen). Zum anderen wollte man dem Vorwurf vorbeugen, man greife in die durch das Grundgesetz geschützten Eigentumsrechte der Aktionäre und Gläubiger der Banken ein. Dass das Grundgesetz für den Schutz des Eigentums auch Grenzen durch die allgemeinen Gesetze vorsieht und dass die durch das Kreditwesengesetz (KWG) vorgenommenen Einschränkungen von Eigentumsrechten dem Schutz der Allgemeinheit vor den möglicherweise von Finanzinstituten ausgehenden Risiken dient, wurde dabei verdrängt. $^{23}$

Das KWG sah durchaus vor, dass die Behörden in Gefahrensituationen die Kontrolle über eine Bank übernehmen könnten, auch wenn die Bank sich noch keiner Verletzung von Liquiditäts- oder Eigen-

21 Zum Krisenverlauf nach dem Lehman-Konkurs siehe Admati und Hellwig a.a.O. (Fn. 15), Kap. 5.

22 Zu diesem Zeitpunkt galt Hypo Real Estate noch als solvent; die Verluste des Bundes bei Hypo Real Estate gehen maßgeblich auf den erst später erfolgten griechischen Schuldenschnitt zurück. 
mittelvorschriften schuldig gemacht hatte. Jedoch wurde diese Möglichkeit entsprechend dem Wortlaut des § 46 KWG eng als Instrument des Gläubigerschutzes interpretiert, derart dass ein solcher Eingriff zu einem Einfrieren von Vermögenswerten führen müsste, ohne Rücksicht auf schädliche Systemwirkungen eines solchen Vorgehens. Das Anfang Oktober 2008 eingebrachte und in wenigen Tagen verabschiedete erste Finanzmarktstabilisierungsgesetz beruhte dann weitgehend auf dem Prinzip, dass der Bund Banken durch Garantien oder Kapitalbeteiligungen stützen würde, ohne dass in die Autonomie der Banken eingegriffen würde.

In der Folge machte man sich an eine Reform der Verfahren zum Umgang mit Banken in Schieflagen. Die vorbereitende Arbeit der Ministerien für das Bankenrestrukturierungsgesetz begann im März 2009; zu diesem Zeitpunkt wurde ich gebeten, einen im Bundesministerium für Wirtschaft vorbereiteten Entwurf zu kommentieren, und merkte an, es fehle nach wie vor an Möglichkeiten, in einer Krise die Kontrolle über ein Institut zu übernehmen, um Schaden von der Allgemeinheit abzuwenden, ohne dass man sich den Automatismen des Insolvenzrechts unterwerfe.

Die Abstimmung zwischen den beteiligten Ministerien (nach der Bundestagswahl 2009 nur noch Finanzen und Justiz) dauerte bis zur Vorlage eines Referentenentwurfs im Juli 2010. Dieser wurde im August 2010 von der Bundesregierung verabschiedet und in die Gesetzgebung eingebracht. Bei der Anhörung des Finanzausschusses des Deutschen Bundestags am 6. Oktober 2010 erklärte ich, der Entwurf biete Verbesserungen in einigen Details, biete aber noch immer kein tragfähiges Verfahren für den Umgang mit Banken in Schieflagen, da wesentliche Schwächen der früheren KWGBestimmungen unverändert beibehalten waren, u.a. die Priorisierung des Gläubigerschutzes gegenüber dem Systemschutz, dies im Unterschied zu z.B. dem Banking Act des Vereinigten Königreichs von $2009 .^{24}$

Am Rande dieser Anhörung erklärte mir ein Mitglied des Deutschen Bundestags, es teile meine kritische Einschätzung, der Bundestag werde aber gar nicht die Zeit haben, sich substanziell mit dem Gesetz auseinanderzusetzen, das Gesetz müsse vor Jahresende verabschiedet werden, da sonst die im Finanzmarktstabilisierungsgesetz von 2008 vorgesehene Frist für die Existenz der FMSA und des SoFFin auslaufe. Das Bankenrestrukturierungsgesetz wurde fast unverändert Anfang Dezember 2010 verabschiedet, übrigens in derselben Sitzung, in der der Präsident des Deutschen Bundestags mit Bezug auf ein anderes Gesetzgebungsverfahren (Verlängerung der Laufzeiten für Atomkraftwerke) kritisierte, dass die Bundesregierung die Gesetzgebungsverfahren so handhabe, dass dem Parlament keine angemessene Zeit zur Beratung bleibe.

Der Entwurf des Bankenrestrukturierungsgesetzes war dem Deutschen Bundestag mit dem Versprechen vorgelegt worden, dieses Gesetz werde verhindern, dass man je wieder in eine Zwangslage kommen werde wie im Herbst 2008 bei Hypo Real Estate. Kaum einen Monat nach der Verabschiedung dieses Gesetzes, im Januar 2011, schrieb das Bundesministerium der Finanzen dem Expertenrat Ausstiegsstrategien, die vom Expertenrat vorgeschlagene Möglichkeit einer Abwicklung der inzwischen vollständig in der Hand des Bundes befindlichen Bank Hypo Real Estate könne nicht in Be-

24 Siehe M. Hellwig, Stellungnahme für die Öffentliche Anhörung des Finanzausschusses des Deutschen Bundestages zum Gesetzentwurf der Bundesregierung „Entwurf eines Gesetzes zur Restrukturierung und geordneten Abwicklung von Kreditinstituten, zur Errichtung eines Restrukturierungsfonds für Kreditinstitute und zur Verlängerung der Verjährungsfrist der aktienrechtlichen Organhaftung (Restrukturierungsgesetz)“ am 6. Oktober 2010, ferner: M. Hellwig, The Problem of Bank Resolution is unsolved: A Critique of the German Bank Restructuring Act of 2010, in: P. Kenadjian (ed.), Too Big to Fail - Brauchen wir ein Sonderinsolvenzrecht für Banken?, Institute for Law and Finance Series, De Gruyter, Berlin, 2012, 35 - 62, und M. Hellwig, a.a.O. (Fn. 13). 
tracht gezogen werden. Es sei nämlich zu befürchten, dass bei Bekanntwerden eines solchen Vorhabens die kurzfristigen Gläubiger des Instituts ihre Mittel abziehen würden und das Institut erneut in eine Liquiditätskrise kommen würde. In diesem Fall habe der Bund keine Mittel, mit einer solchen Krise umzugehen. Abgesehen davon, dass, wie noch zu erläutern sein wird, die Aussage des Ministeriums sachlich falsch war, fand ich es schockierend, dass eben das Ministerium, das maßgeblich mitverantwortlich war für das Bankenrestrukturierungsgesetz mit dem Versprechen, man werde nie mehr in eine Zwangslage wie 2008 kommen, nunmehr behauptete, man werde mit einer Liquiditätskrise einer systemrelevanten Bank ebenso wenig umgehen können wie im Herbst 2008. ${ }^{25}$

Die Mängel des Bankenrestrukturierungsgesetzes traten auch zutage, nachdem der EU-Gipfel im Oktober 2011 eine Erhöhung der Eigenkapitalanforderungen für systemrelevante Banken beschlossen hatte. Es stellte sich nämlich heraus, dass das Gesetz keine Handhabe zur Umsetzung dieses Beschlusses bot. Dafür sorgte erst das 2. Finanzmarktstabilisierungsgesetz von 2012, eingebracht mit der Begründung, diesmal gehe es um die Abwehr einer Systemkrise und nicht mehr um die Schieflage einer einzelnen Bank, wofür man schon durch das Gesetz von 2010 Vorsorge getroffen habe - als ob der Fall Hypo Real Estate vom Oktober 2008, der 2010 als Grund für das Bankenrestrukturierungsgesetz genannt wurde, nichts mit einer Systemkrise zu tun gehabt hätte und als ob es nicht 2012 wie 2010 um die Regelung der Eingriffsmöglichkeiten der Aufsicht gegenüber den jeweils einzelnen Banken gegangen wäre. Beim 3. Finanzmarktstabilisierungsgesetz, das die Frist für ein Auslaufen der Geltung des 2. Finanzmarktstabilisierungsgesetzes aufhob, ersparte man sich eine derartige Begründung und verwies lediglich darauf, dass man die Regelung übergangsweise bis zur Umsetzung der europäischen Bankensanierungs- und -abwicklungsrichtlinie (Bank Recovery and Resolution Directive - BRRD) benötige. ${ }^{26}$

Zurück zum Vorschlag des Expertenrats Ausstiegsstrategien: Der Expertenrat legte dem Bundesministerium der Finanzen am 31. Dezember 2010 einen ersten Entwurf seines Berichts vor. Dieser enthielt die Empfehlung, im Falle der Hypo Real Estate und im Falle der West LB die Möglichkeit eines „Ausstiegs“ vermittels einer Abwicklung der Bank zu prüfen. Die Empfehlung gründete sich auf die Einschätzung, dass, wie oben (Tzz. 15, 16) ausgeführt wurde, es in bestimmten Teilen des deutschen Finanzsystems Überkapazitäten gebe, mit der Folge einer allzu hohen Wettbewerbsintensität, die die Banken zum Eingehen übermäßiger Risiken veranlasst habe. Der Expertenrat hielt es für gefährlich, wenn die staatlichen Stützungsmaßnahmen einen Abbau der Überkapazitäten verhinderten. Der Expertenrat zog auch in Betracht, dass eine vollständige Abwicklung der HRE durch die FMSA insofern von Vorteil wäre, als die Abwicklung nicht unter Zeitdruck erfolgen würde und die Refinanzierung zu den dem Bund zur Verfügung stehenden Konditionen sehr günstig sein würde.

25 Dazu siehe auch M. Hellwig (2014), Stellungnahme für den Finanzausschuss des Deutschen Bundestags zum Entwurf eines Gesetzes zur Umsetzung der Richtlinie 2014/59/EU des Europäischen Parlaments und des Rates vom 15. Mai 2014 zur Festlegung eines Rahmens für die Sanierung und Abwicklung von Kreditinstituten und Wertpapierfirmen (BRRD-Umsetzungsgesetz), https://www.bundestag.de/blob/ 333188/2466b19bce1a95b29b00f6c8f63f5289/09---prof--hellwig-data.pdf.

26 Aus meiner Stellungnahme von 2014 (Fn. 25) wird deutlich, dass ich auch die von der BRRD vorgesehenen Verfahren nicht für tragfähig halte, zum einen weil die Anwendung des Verfahrens auf grenzüberschreitend systemrelevant tätigen Banken solche systemrelevanten Tätigkeiten beendet, bei denen die unternehmerischen Prozesse über alle Töchter der Institution hinweg integriert werden, z.B. über ITSysteme, zum anderen weil es keine Mechanismen gibt, die die Liquiditätsversorgung während des Verfahrens sicherstellen würden. Im Kontext der Stellungnahme für das Bundesverfassungsgericht geht es mir aber nicht um die Frage, ob die Reformen genügen, sondern um den Umgang der Bundesregierung mit den Gesetzgebungsverfahren und die Diskrepanz zwischen dem, was von den Gesetzesvorlagen behauptet wird, und dem, was die Regelungen tatsächlich leisten. 
Ministerium und FMSA zogen es vor, die Hypo Real Estate bzw. die Deutsche Pfandbriefbank pbb für eine Privatisierung vorzubereiten (die 2015 tatsächlich stattfand). Man verwies auf das Gutachten einer Beratungsfirma, wonach dies die kostengünstigste Strategie wäre. Das Gutachten enthielt allerdings einen Denkfehler: Die Gebühren, die die Bank der FMSA zu zahlen hatte, wurden als Kosten behandelt. Das fiel ins Gewicht, denn bei einer langsamen Abwicklung des Gesamtbestands an Wertpapieren der Bank würden solche Gebühren während vielen Jahren zu zahlen sein. Dabei wurde allerdings übersehen, dass diese Gebühren nur aus Sicht der Bank Kosten waren, nicht aber aus Sicht des Bundes, der gleichzeitig Eigentümer der Bank und Träger der FMSA war. Der Expertenrat Ausstiegsstrategien wies auf diesen Denkfehler hin, machte aber die Erfahrung, dass weder die FMSA noch das Bundesministerium der Finanzen bereit waren, sich auf eine Diskussion darüber einzulassen.

Im Übrigen war das Gutachten, auf das das Ministerium und die FMSA sich stützten auch nicht darauf eingegangen, dass ein Verbleib der HRE/pbb im Markt die Möglichkeiten von Commerzbank und WestLB, die Pfandbrieftöchter Eurohypo und Westimmo zu verkaufen, deutlich schmälern musste, was wiederum Einfluss auf den Wert der Beteiligungen des Bundes an diesen Instituten haben musste.

Das Ministerium forderte den Expertenrat, wie erwähnt, auf, die genannten Empfehlungen zu ändern. Der Expertenrat ging darauf nicht ein. Zur Warnung, bei Ankündigung einer Strategie der Abwicklung der Hypo Real Estate/pbb könne die Refinanzierung der Bank zusammenbrechen, führte der Expertenrat stattdessen aus, das Problem werde sich erübrigen, wenn die Bank sämtliche Vermögenswerte in die FMS-Wertmanagement einbringe, die von der FMSA verwaltete „Bad Bank“, in die die Hypo Real Estate schon vorher, im Herbst 2010, Vermögenswerte mit einem Nennwert von insgesamt $€ 170$ Mrd. eingebracht hatte. Die Refinanzierung werde dann Aufgabe der FMSA sein, die als Einrichtung des Bundes keine Probleme damit haben werde.

Das Ministerium nahm auch Anstoß an den Ausführungen des Expertenrats zum Übermaß an Fristentransformation durch Pfandbriefbanken, konkret an der Aussage, die Finanzierung der Überdeckung von Pfandbriefen (s.o. Tz. 15) über den Geldmarkt, sei ein Indiz für Fehlanreize, derart langfristige Anlagen sollten langfristig finanziert werden, z.B. durch Lebensversicherer, die selbst langfristige Verbindlichkeiten hätten und daher besser in der Lage wären, die mit langfristigen Anlagen einhergehenden Bewertungsrisiken zu tragen. Diese Aussage, so das Ministerium, stehe im Widerspruch zur Strategie der Bundesregierung, die Versicherer zu einer besseren Kontrolle der von ihnen eingegangenen Risiken anzuhalten. Bei der Wahl der Laufzeit der Anlagen, in die man investiert, sind allerdings immer zwei Risiken zu bedenken, bei langfristigen Anlagen das bei einem Verkauf vor Fälligkeit relevante Kursrisiko und bei kurzfristigen Anlagen das bei einem langfristigen Anlagehorizont maßgebliche Risiko, dass eine Wiederanlage nur zu weniger günstigen Zinssätzen möglich sein könnte. Dem Ministerium war offensichtlich nicht bewusst, dass für einen Lebensversicherer oder einen Rentenversicherer mit langfristigen Verbindlichkeiten letzteres Risiko stärker ins Gewicht fällt als ersteres, und dass die u.a. mit Unterstützung von Bundesregierung und Aufsicht in den vergangenen zwanzig Jahren geförderte Entwicklung der Anlagestrategien im Versicherungssektor hin zu kürzeren Laufzeiten für diesen Sektor selbst sehr gefährlich ist. Der Expertenrat ist auch dieser Aufforderung des Ministeriums nicht gefolgt, sondern hat stattdessen seine Überlegungen noch weiter ausgeführt und begründet. ${ }^{27}$

Ich halte die hier beschriebenen Vorgänge für problematisch. Damit meine ich nicht den Umstand, dass die Bundesregierung in vielen Punkten anders vorgegangen ist, als ich es für richtig gehalten hätte. Ich meine vielmehr den Umstand, dass eine substantielle öffentliche Diskussion zu wesentlichen 
Punkten gar nicht stattgefunden hat, dass die Exekutive ihren Einfluss auf die Verfahrensgestaltung der Legislative benutzt hat, um die eingehende materielle Auseinandersetzung mit den Gesetzesvorlagen zu behindern, dass sie ihre eigenen Vorstellungen in großen Teilen nicht der Prüfung durch eine öffentliche Diskussion unterworfen hat und dass es nach wie vor keine inhaltliche Aufarbeitung des Umgangs der deutschen Institutionen mit den Krisen, ihren Ursachen und den Maßnahmen zu ihrer Bewältigung gibt.

\section{Transparenz und Finanzstabilität}

Nach der ausführlichen Behandlung des Kontextes der seinerzeitigen parlamentarischen Anfragen und ihrer Beantwortung durch die Bundesregierung komme ich zurück zum eigentlichen Gegenstand der Anfrage des Bundesverfassungsgerichts. Was lässt sich über eine mögliche Gefährdung der Funktionsfähigkeit der Finanzmarktaufsicht und des Erfolgs der staatlichen Stützungsmaßnahmen durch die genannten Anfragen sagen? Die Feststellung, dass das Argument einer Gefährdung der Finanzstabilität gegenüber dem Expertenrat Ausstiegsstrategien fehlerhaft eingesetzt wurde, erübrigt nicht eine allgemeine Auseinandersetzung mit diesem Argument, insbesondere im Zusammenhang der konkreten Anfragen, um die es hier geht.

\subsection{Vertraulichkeitsgebote}

Vorab einige Bemerkungen zum Recht der beaufsichtigten Banken auf Diskretion der Aufsicht und zum Recht der Vertragspartner auf Wahrung der vereinbarten Vertraulichkeit. Beide Rechte sind ernst zu nehmen, aber beide Rechte können auch missbraucht werden.

Ein Anspruch des Käufers der IKB auf Vertraulichkeit über den Kaufpreis mag sich aus dem Vertrag ergeben, aber man fragt sich, warum die Bundesregierung bzw. ihre Vertreter dieser Vertragsklausel zugestimmt haben. In Anbetracht der Diskrepanz zwischen dem ursprünglich erhofften und dem in der Presse kolportierten Preis könnte ich mir sogar vorstellen, dass das Interesse des Verkäufers an dieser Vertraulichkeit größer war als das des Käufers. ${ }^{28}$ Jedenfalls ist die Vertraulichkeit hier kein Korrelat eines hoheitlichen Eingriffs in die Autonomie des Betroffenen.

Im Verhältnis zwischen Aufsicht und Banken dagegen ist der Anspruch der Banken auf Diskretion der Aufsicht ein Korrelat des Umstands, dass die Aufsicht hoheitliche Rechte geltend macht. Eine Veröffentlichung der von der Aufsicht gewonnenen Informationen könnte der Bank in ihren Beziehungen mit Geldgebern und Wettbewerbern schaden und würde die Schwere des hoheitlichen Eingriffs unverhältnismäßig vergrößern. Die Erwartung einer solchen Veröffentlichung würde auch das Verhalten der Bank gegenüber der Aufsicht belasten. Dadurch könnte die Arbeit der Aufsicht deutlich erschwert werden.

Allerdings habe ich beobachtet, dass Aufsichtsbehörden sich auch dort auf das Gebot der Vertraulichkeit berufen, wo ein Austausch von Informationen unproblematisch erscheint. Im Rahmen des Europäischen Systemrisikoausschusses beispielsweise kommt es immer wieder vor, dass die in der europäischen Verordnung gebotene Pflicht zum Informationsaustausch der nationalen Behörden untereinander

28 Unter Berufung auf einen Bericht der Süddeutschen Zeitung nennt Kaserer, a.a.O. (Fn. 5) einen Verkaufspreis von ca. $€ 100$ Mio. 
und mit dem Europäischen Systemrisikoausschuss unter Berufung auf Gebote der Vertraulichkeit verletzt wird oder aber erst nach langen Verhandlungen erfüllt wird - dies, obwohl die Verantwortlichen der anderen Behörden und des Europäischen Systemrisikoausschusses selbst denselben Geheimhaltungspflichten unterliegen. Die Fähigkeit des Europäischen Systemrisikoausschusses, sich aufbauende Risiken für das europäische Finanzsystem insgesamt frühzeitig zu erkennen, wird dadurch deutlich beeinträchtigt.

Im Übrigen wäre zu fragen, inwiefern eine Bank, die auf staatliche Stützungsmaßnahmen angewiesen ist, denselben Anspruch auf Vertraulichkeit stellen kann wie eine andere Bank. Im vorliegenden Verfahren betreffen die Fragen nach einzelnen Banken fast durchweg solche Banken, die von der Krise von 2007-2009 ernsthaft betroffen waren und staatlicher Hilfe bedurften. Derartige Hilfen waren und sind in unserer Wirtschafts- und Rechtsordnung eigentlich nicht vorgesehen. Die Frage, warum diese Banken in solche Schwierigkeiten gekommen waren und wie das Verhalten der Aufsicht und der Banken im Vorfeld der Krise zu beurteilen ist, war und ist von großem öffentlichem Interesse. Soweit die betroffenen Banken eine Schädigung ihres Verhältnisses zu Geldgebern und Wettbewerb geltend machen, ist dagegen zu halten, dass diese Banken gerade in ihren Beziehungen zu Geldgebern und Wettbewerbern zunächst einmal massiv von der staatlichen Unterstützung profitiert haben.

In einem Fall wie dem der in Nr. 10 und Nr. 12 der Kleinen Anfrage vom 11. November 2010 angesprochenen Beteiligung der BayernLB an der österreichischen Hypo Group Alpe-Adria (HGAA) ist das Vertraulichkeitsargument der Bundesregierung besonders problematisch. Der 2014 erstellte Bericht der österreichischen Untersuchungskommission zu HGAA erweckt den Eindruck, dass diese Akquisition, die den Freistaat Bayern mehrere Milliarden Euro kostete, ohne angemessene due diligence zustande gekommen ist. ${ }^{29}$ Die Antwort der Bundesregierung auf die genannten Fragen betont die unternehmerische Autonomie und „das berechtigte Interesse“ der Bank an der Vertraulichkeit der Entscheidungsprozesse. Wenn tatsächlich die bei einer solchen Akquisition zu beachtenden Sorgfaltsregeln verletzt wurden, sehe ich nicht, warum das Interesse an der Geheimhaltung dieses Tatbestands „berechtigt“ sein sollte, auch nicht, warum die unternehmerische Autonomie der BayernLB einer Einforderung angemessener due diligence durch die Aufsicht entgegenstehen sollte. Der Fall weist Parallelen auf zu der 2007/2008 vorgenommenen Akquisition der italienischen Bank Antonveneta durch die Bank Monte dei Paschi di Siena, ebenfalls ohne angemessene due diligence und zu einem deutlich überhöhten Preis; diese Akquisition hat maßgeblich zu den Solvenzproblemen von Monte dei Paschi beigetragen und wird in Deutschland gern als Beispiel für skandalöse Nachlässigkeit der Aufsicht unter der Präsidentschaft von Mario Draghi bei der Banca d’Italia kritisiert.

Im Umgang mit dem Gebot des Vertrauensschutzes und des Schutzes der Wettbewerbsfähigkeit der betroffenen Banken sollte klar unterschieden werden zwischen dem Normalfall einer Bank, die ohne Schwierigkeiten und ohne staatliche Unterstützung das Auf und Ab ihrer Geschäftstätigkeit meistert, und dem Sonderfall einer Bank, die das nicht kann und die staatliche Hilfe in Anspruch nimmt. Das Bedenken, die Aussicht auf eine Veröffentlichung von Information könnte das Verhalten der Bank gegenüber der Aufsicht belasten, betrifft den Normalfall. Dieses Bedenken für den Sonderfall einer Bank geltend zu machen, die staatliche Hilfe in Anspruch nimmt, ist nicht vereinbar mit der Norm, dass es diesen Sonderfall eigentlich gar nicht geben dürfte.

29 Bericht der unabhängigen Untersuchungskommission zur transparenten Aufklärung der Vorkommnisse rund um die Hypo Group Alpe-Adria, Wien, 2. Dezember 2014,

http://cdn.untersuchungskommission.at/pdf/BerichtHypo-Untersuchungskommission.pdf, abgerufen am 3. Juni 2017. 
Im Übrigen ist anzumerken, dass in den USA bestimmte Informationen, z.B. über Beanstandungen der Aufsicht, grundsätzlich mit einer gewissen Verzögerung im Nachhinein publiziert werden, auch wenn es keine staatlichen Hilfsmaßnahmen o.ä. gegeben hat. Dort gilt das Prinzip, dass Transparenz, auch über bestimmte Schwierigkeiten von Banken, der Finanzstabilität und insofern im öffentlichen Interesse liegt, da die Geschäftspartner der Bank die Risiken, die sie eingehen, um so besser einschätzen können, je besser sie selbst informiert sind.

\subsection{Gefahren für die Stabilität der betroffenen Banken?}

Die Antwort der Bundesregierung auf Nr. 6 der Kleinen Anfrage vom 11. November 2010 macht weiterhin geltend, dass eine Veröffentlichung der Information, wann bzw. wie oft bei einzelnen Instituten Sonderprüfungen der Aufsicht stattgefunden haben, einen irreversiblen Vertrauensverlust in das einzelne Institut mit entsprechenden Reaktionen der Märkte, insbesondere der Gläubiger begründen könnte.

Es trifft sicher zu, dass negative Information über ein Institut solche Reaktionen hervorrufen kann. So waren 2011 die Reaktionen der Gläubiger auf die Information über die voraussichtliche Höhe des griechischen Schuldenschnitts bei der belgisch-französischen Bank Dexia besonders ausgeprägt. Aufgrund der sonstigen zur Verfügung stehenden Informationen war abzusehen, dass diese Bank durch den griechischen Schuldenschnitt in die Insolvenz getrieben werden würde.

Jedoch ist die Mitteilung über die Frequenz von in der Vergangenheit vorgenommenen Sonderprüfungen von anderer Qualität als die Mitteilung über in Aussicht stehende Verluste einer Bank aus einer größeren Anlage. Im vorliegenden Fall ging es um Sonderprüfungen in den Jahren 2005 bis 2008. Als Banken genannt wurden: Bayern LB, Sachsen LB, West LB, HSH Nordbank, IKB Deutsche Industriebank, Düsseldorfer Hypothekenbank, Hypo Real Estate - HRE, Commerzbank und Dresdner Bank. Von diesen Banken waren Sachsen LB, IKB, Hypo Real Estate und Dresdner Bank gar nicht mehr als selbständige Banken tätig. Bayern LB, West LB, HSH Nordbank und Commerzbank hatten umfängliche Kapitalhilfen und Garantien der Eignerländer bzw. des Bundes erhalten. Es ist nicht ersichtlich, dass eine Information über die Zahl der in den Jahren 2005 bis 2008 von der BaFin vorgenommenen Sonderprüfungen im Jahre 2010 den Anlegern im Markt irgendeine relevante zusätzliche Information vermittelt hätte: Dass diese Banken in der Krise Probleme gehabt hatten, war bekannt. Ob diese Probleme noch fortbestehen würden, hing davon ab, ob die Stützungsmaßnahmen des Bundes und der Länder ausreichen würden. Solange die Anleger davon ausgehen konnten, dass die Stützungsmaßnahmen von Bund und Ländern nachhaltig fortgesetzt werden würden, gab es keinen Grund für sie, die Refinanzierung dieser Institute zu schmälern. Für die Beantwortung dieser Frage jedoch gab und gibt die Information über die Frequenz der Sonderprüfungen in den Jahren vor der Krise nichts her.

In diesem Kontext ist zu erwähnen, dass die BaFin Anfang 2013 eine Sonderprüfung für Schiffskredite ankündigte. Diese Ankündigung betraf insbesondere HSH Nordbank, Commerzbank und Nord LB, von denen bekannt war, dass sie sehr viele Schiffskredite vergeben hatten. Es ist nicht ersichtlich, dass die Ankündigung der BaFin zu Vertrauensverlusten bei den Gläubigern dieser Banken geführt hätte. Die im Frühjahr 2013 angekündigte Wiedererhöhung der Zweitverlustgarantie der Länder Hamburg und Schleswig-Holstein für HSH Nordbank (von $€ 7$ Mrd. auf $€ 10$ Mrd.) wurde nicht etwa damit begründet, dass die voraussichtlichen Verluste der Bank bei Schiffskrediten, geschweige denn die Sonderprüfung der BaFin, das Vertrauen der Gläubiger erschüttert hätten; das erklärte Ziel war vielmehr eine Reduktion der den Vermögensanlagen der Bank zugeschriebenen Risiken und damit eine Reduktion der erforderlichen Eigenmittel der Bank. 
In der wirtschaftstheoretischen Literatur über Banken spielt das Phänomen des „Bank Runs“ oder Schaltersturms eine große Rolle. Da die Bank zumeist illiquide Titel hält, etwa Unternehmenskredite, sich gleichzeitig aber mit kurzfristig abziehbaren Einlagen bzw. kurzfristigen Geldmarktkrediten finanziert, besteht rein theoretisch immer das Risiko, dass alle Geldgeber gleichzeitig ihre Mittel abziehen wollen und die Bank diesen ihren Verpflichtungen nicht nachkommen kann. Wenn nun die Geldgeber erwarten, dass die anderen Geldgeber ihre Mittel abziehen wollen, so werden sie selbst sich an diesem Schaltersturm beteiligen wollen, d.h. in der Sprache der Theorie strategischer Spiele, entspricht es einem Gleichgewichtsverhalten, dass alle gleichzeitig ihre Mittel abziehen wollen. ${ }^{30}$ Rein theoretisch kann man sich auch vorstellen, dass eine Verstärkung der Aktivität von Sonnenflecken einen solchen Schaltersturm auslöst - oder irgendeine andere Information, ob diese Information nun inhaltlich bedeutsam ist oder nicht. ${ }^{31}$

Allerdings steht die praktische Bedeutung dieser Überlegungen in keinem Verhältnis zu dem intellektuellen Kitzel, der auf der Vorstellung beruht, dass eine Bank ohne „richtigen Grund“, nur aufgrund sich selbst bestätigender Erwartungen der Beteiligten über das Verhalten der jeweils anderen, zusammenbrechen könnte. Die empirische Literatur zu Bankzu-sammenbrüchen zeigt ziemlich eindeutig, dass Schalterstürme nicht aus heiterem Himmel kommen, sondern durch neue und vor allem schwerwiegende Informationen ausgelöst werden, die die Fähigkeit der Institute zur Erfüllung ihrer Verbindlichkeiten infrage stellen. ${ }^{32}$

Die Einschätzung, dass Schalterstürme durch substantielle Informationen ausgelöst werden, führt nicht zu der Empfehlung, solche Informationen unter der Decke zu halten. Im Gegenteil, ein Teil der wirtschaftstheoretischen Literatur ist der Auffassung, es sei wichtig, dass auch negative Information bekannt gemacht wird. Wenn das Bekanntwerden solcher Information negative Folgen für die Bank haben könne, so werde die Furcht vor eben diesen Folgen dazu beitragen, dass die Manager der Banken angemessene Verhaltensanreize bekommen. ${ }^{33}$ Ich bin bezüglich dieser Anreizwirkungen skeptisch, ${ }^{34}$ teile aber die Auffassung, dass negative Information sich nicht auf Dauer unter der Decke halten lässt und dass schon der Versuch der Verheimlichung gefährlich sein kann, da er mit einer Verschleppung

30 D. Diamond und P. Dybvig, Bank Runs, Deposit Insurance and Liquidity, Journal of Political Economy 91 (1983), 401-419.

31 Die neuere wirtschaftstheoretische Literatur geht über dieses einfache Modell des Schaltersturms als Ergebnis von sich selbst bestätigenden Erwartungen über das Verhalten der jeweils anderen hinaus und untersucht auch den Einfluss von neuer Information, so etwa I. Goldstein und A. Pauzner, Demand Deposit Contracts and the Probability of a Bank Run, Journal of Finance 60 (2005), 1293-1327, unter Rückgriff auf S. Morris und H. Shin, Unique Equilibrium in a Model of Self-Fulfilling Currency Attacks, American Economic Review 88 (1998), 587 - 597. Der Beitrag dieser Literatur besteht darin, zu zeigen, dass eine Multiplizität möglicher Gleichgewichte ausgeschlossen ist, wenn die Informationen der verschiedenen Anleger hinreichend unabhängig voneinander sind. Auch in diesen Modellen besteht allerdings die Möglichkeit, dass die Reaktion des Systems auf geänderte Information unverhältnismäßig hart ausfallen kann.

32 Für einen Überblick siehe C. Calomiris und G. Gorton, The Origins of Banking Panics: Models, Facts and Bank Regulation, in: G. Hubbard (ed.), Financial Markets and Financial Crises, University of Chicago Press, Chicago 1991, 109 - 173. C. Calomiris und J. Mason, Fundamentals, Panics, and Bank Distress During the Depression, American Economic Review 93 (2003), 1615-1647, und I. Schnabel, The German Twin Crisis of 1931, Journal of Economic History 64 (2004), 822 - 871, zeigen, dass dieses Prinzip auch für die Schalterstürme in der Großen Weltwirtschaftskrise galt: Sowohl in den USA als auch in Deutschland wussten die Gläubiger sehr genau zwischen den einzelnen Banken zu unterscheiden, je nachdem, ob schädliche neue Information sie betraf oder nicht.

33 So etwa D. Diamond und R. Rajan, Liquidity Risk, Liquidity Creation and Financial Fragility, Journal of Political Economy 109 (2001), 287-327.

34 Siehe A. Admati und M. Hellwig, Does Debt Discipline Bankers? An Academic Myth About Bank Indebtedness, Stanford Discussion Paper 2013, http://bankersnewclothes.com/wp-content/uploads/ 2016/12/Omitted-chapter-academic-myth.pdf , aufgerufen am 8. Mai 2017. 
der ggfs. erforderlichen Korrekturmaßnahmen einhergeht. Die empirische Literatur zu Bankenkrisen zeigt deutlich, dass die letztlich nicht zu vermeidende Bereinigung von Krisen für die Volkswirtschaft und für den Staatshaushalt um so teurer ausfällt, je länger man sie vertuscht und die Bereinigung hinausschiebt. $^{35}$

\subsection{Transparenz als Steuerungsinstrument}

Die Einschätzung, dass Transparenz grundsätzlich erwünscht ist, gehört zu den Grundlagen der Bankenregulierung nach den Basler Abkommen zur Bankenaufsicht. Als sogenannte „dritte Säule“ der Bankenaufsicht nennen die Basler Abkommen die Förderung von Transparenz über laufende Entwicklungen als Grundlage für eine Disziplinierung der Banken durch die Märkte für Aktien und Schuldtitel der Banken.

Diese Einschätzung begründet auch das Prinzip der Bilanzierung nach dem Zeitwert (fair value accounting). Hier wird die Anpassung der Bilanzbewertungen an laufende Entwicklungen gefordert, damit die Anleger im Markt jeweils bestmöglich über die Situation der Bank informiert sind.

Im Oktober 2008 wurde das Prinzip der Bilanzierung nach dem Zeitwert ausgesetzt, weil es zur Krisendynamik beizutragen schien. ${ }^{36}$ Bewertungsanpassungen zwangen die Banken, Verluste zu verbuchen, die ihr Eigenkapital minderten, so dass sie die regulatorischen Eigenkapitalanforderungen nicht mehr erfüllten und weitere Vermögenswerte verkaufen mussten, was wiederum auf die Kurse drückte. Die wissenschaftliche Literatur zu dieser Episode kommt allerdings zu dem Schluss, dass der Fehler nicht so sehr beim Zeitwertprinzip als solchem liege als vielmehr in der Verbindung des Zeitwertprinzips mit einer bestimmten Form der Eigenkapitalregulierung. ${ }^{37}$

Bei Vermögenswerten im Handelsbuch der Bank ist die Anwendung des Zeitwertprinzips insofern unproblematisch, als es jeweils Marktpreise gibt, an denen man sich orientieren kann. Bei Vermögenswerten im Kreditbuch der Bank ist das zumeist nicht möglich, da es für die Unternehmenskredite keine Märkte gibt. Bei diesen Titeln ist die angemessene Bewertung eine Frage der Einschätzung der Wahrscheinlichkeit, dass der Kreditnehmer am Ende (doch noch) zahlen wird. Für das Management der Bank und für die Aufsicht der Bank ist dabei die Versuchung groß, so tun, als gäbe es kein Problem. Kurzfristig ist das auch oft die angemessene Reaktion. Jedoch sind die Anreize beider verzerrt, so dass es auch zu einem Übermaß an „Geduld“ kommen kann, das volkswirtschaftlich hohe Kosten verursacht. $^{38}$ So wird zum Beispiel das Nachlassen des Produktivitätswachstums in Japan in den 1990er Jahren darauf zurückgeführt, dass große japanische Banken de facto insolvent waren, die Insolvenz aber nicht offen gelegt wurde, da große, de facto ebenfalls insolvente Kreditnehmer dieser Banken nicht in den Konkurs geschickt wurden; das Fortbestehen dieser Unternehmen, noch dazu mit einem Rückhalt durch zahlungsfähige, wenn auch überschuldete Banken, hemmten den Wettbewerb durch neue Unternehmen, die zur Produktivitätssteigerung hätten beitragen können. ${ }^{39}$ Auch diese Erfahrung spricht dagegen, dass man versucht, relevante Information unter der Decke zu halten, um Unruhe zu vermeiden.

35 Siehe Advisory Scientific Committee of the European Systemic Risk Board, Forbearance, Resolution and Deposit Insurance, Report 01/2012, Tz. 33, und die dort angegebene Literatur.

36 Siehe z.B. A. Admati und M. Hellwig, a.a.O. (Fn. 16), Kap. 5.

37 So vor allem C. Laux und C. Leuz, Did Fair Value Accounting Contribute to the Financial Crisis?, Journal of Economic Perspectives 24 (1) (2010), 93 - 118.

38 Advisory Scientific Committee a.a.O. (Fn. 34).

39 Siehe T. Hoshi und A. Kashyap, Japan's Financial Crisis and Economic Stagnation, Journal of Economic Perspectives 18 (1) (2004), 3 - 26. 
Die Auffassung, dass Transparenz grundsätzlich wünschenswert und der Finanzstabilität zuträglich ist, ist in jüngster Zeit durch einige Autoren bestritten worden. ${ }^{40}$ Diese Autoren betonen den Unterschied zwischen Aktien und geldähnlichen Titeln und verweisen darauf, dass Aktien „informationssensitiv“ sind, geldähnliche Titel aber nicht. Gemeint ist, dass die Erträge auf Aktien und die Bewertung von Aktien im Detail von den Geschäftsergebnissen der betreffenden Unternehmen abhängen, dass diese Abhängigkeit Anreize schafft, Ressourcen aufzuwenden, um die betreffende Information zu beschaffen, und dass dieser Mechanismus die Funktionsfähigkeit der Märkte stört, da jeder Marktteilnehmer fürchten muss, in einer konkreten Transaktion mit jemandem zu tun zu haben, der besser informiert ist, und ihn übervorteilt. Bei geldähnlichen Titeln dagegen sind die Forderungen des Inhabers unabhängig vom Geschick der Emittenten, so dass Information über den Emittenten keinen Einfluss hat auf die Bedingungen, unter denen diese Titel gehandelt werden; diese „Informationsinsensitivität“ erhöht die Handelbarkeit der Titel und macht sie zu guten Substituten für Bargeld. Das Paradebeispiel sind Schecks, d.h. Zahlungsanweisungen, die auf Verpflichtungen einer Bank gegenüber dem Inhaber einer Einlage beruhen; in früheren Zeiten wären hier auch Banknoten, d.h. Ansprüche auf Zahlung bestimmter Beträge in Gold zu nennen gewesen. Nach Auffassung dieser Literatur sind Asset-backed commercial paper und Repo-Kredite, d.h. besicherte Geldmarktkredite, die moderne Version dieser früheren geldähnlichen Titel. Damit die Titel liquide bleiben und die Zahlungsmechanismen nicht zerstört werden, sei es notwendig, Information geheim zu halten, so etwa die Information über die Qualität der Sicherheiten.

Ich stehe dieser Literatur kritisch gegenüber. Die Grundthese, dass „informationsinsensitive“ Titel leichter handelbar sind als „informationssensitive“, halte ich für richtig. Wenn der mit einem Titel verbundene Anspruch fest gegeben ist und die Erfüllung dieses Anspruchs nicht von zusätzlichen Bedingungen abhängt, so brauchen die Beteiligten keine Sorge zu haben, was denn der Anspruch am Ende wohl wert sein mag. Diese Eigenschaft hängt aber nicht so sehr davon ab, dass man über den Emittenten nichts weiß, sondern davon, dass es sich um einen Schuldtitel handelt, dessen Inhalt fest vorgegeben ist und von dem man glaubt, dass der Emittent ihn erfüllen kann. Die Fähigkeit von Banken, „geldähnliche Titel“ zu produzieren, hängt m.E. nicht so sehr davon ab, dass die Vielzahl der vergebenen Kredite es unmöglich machen würde, die Bonität der Bank genau zu beurteilen, als vielmehr davon, dass die Vielzahl der vergebenen Kredite eine gewisse Risikodiversifizierung mit sich bringt, was zur Folge hat, dass bei genügender Eigenkapitalausstattung der Bank die emittierten Schuldtitel, auch Einlagen und Banknoten, wenig riskant sind. ${ }^{41}$

Die Übertragung dieser Idee auf besicherte Geldmarktkredite und die Notwendigkeit, Information über die Sicherheiten geheim zu halten, ist nicht überzeugend, zumal die dabei wirkenden Anreizverzerrungen übersehen werden, z.B. die Erwägung, dass ein Repo-Kredit dem Geldgeber und der Bank die Möglichkeit gibt, durch eine Erhöhung der Verschuldung der Bank bei Aussonderung der Sicherheiten die Position der Altgläubiger der Bank zu schädigen. ${ }^{42}$

40 Insbesondere B. Holmström, Understanding the role of debt in the financial system, BIS Working Paper No. 479, Bank für Internationalen Zahlungsausgleich, Basel, 2015, http://www.bis.org/publ/ work479.htm , abgerufen am 3. Juni 2017, sowie T.V. Dang, G. Gorton, B. Holmström, Banks as Secret Keepers, American Economic Review 107 (2017), 1005 - 1029.

41 D. Diamond, Financial Intermediation and Delegated Monitoring, Review of Economic Studies 51 (1984), 393-414, M. Hellwig, Banking, Financial Intermediation, and Corporate Finance, in: A. Giovannini and C. Mayer (eds.), European Financial Integration, Cambridge University Press 1991, 35-63.

42 Siehe A. Admati und M. Hellwig a.a.O. (Fn. 16), Kap. 10, sowie A. Admati, P. DeMarzo, M. Hellwig, P. Pfleiderer, The Leverage Ratchet Effect, Preprint 2013/13, Max Planck Institute for Research on Collective Goods, Bonn, erscheint in: Journal of Finance. 
Im vorliegenden Kontext stellt sich vor allem die Frage, wie mit der Möglichkeit umzugehen ist, dass die „informationsinsensitiven“ Schuldtitel der Bank plötzlich „informationssensitiv“ werden. Dies geschieht, wenn die Risikodiversifizierung nicht erfolgreich war, Verluste das Eigenkapital der Bank angreifen und die Solvenz der Bank infrage gestellt ist. In einer solchen Situation haben die Manager der Bank - und möglicherweise auch die Aufsicht - ein Interesse daran, die Verluste geheimzuhalten, dies in der Hoffnung, dass das Schicksal sich vielleicht noch einmal zum Besseren wendet. Mit dieser Situation hat sich die hier angesprochene Literatur allerdings nicht befasst. Ich bleibe daher bei der Einschätzung, dass es in solchen Situationen besser ist, die Probleme offenzulegen und zu lösen und sie nicht lange schwelen zu lassen.

\subsection{Gefahren für das Finanzsystem?}

Zum Abschluss gehe ich auf die möglichen Auswirkungen einer Veröffentlichung der in Rede stehenden Informationen auf das Finanzsystem als Ganzes ein. Die Bundesregierung verweist auf die Gefahr von Ansteckungseffekten. Tatsächlich ist die Gefahr von Ansteckungseffekten ein wichtiger Grund für die Tätigkeit der staatlichen Aufsicht. Systemwirkungen von Schieflagen oder gar Krisen einzelner Banken können großen volkswirtschaftlichen Schaden anrichten. Im einzelnen sind folgende Typen von Ansteckungseffekten zu unterscheiden: ${ }^{43}$

- $\quad$ Dominoeffekte aufgrund von Zahlungsausfällen, so etwa die Wirkung des LehmanKonkurses auf den Geldmarktfonds Reserve Primary,

- $\quad$ Dominoeffekte aufgrund eines Fortfalls der Institution als potentieller Vertragspartner, so etwa die Wirkung der Mittelabzüge bei Geldmarktfonds auf Verfügbarkeit von Geldmarktfinanzierungen für Hypo Real Estate im September 2008,

- $\quad$ Marktwirkungen der defensiven Maßnahmen von Banken in Schwierigkeiten, so etwa die Kursstürze im September 2008 als Folge des Versuchs von Banken, sich durch den Verkauf von Wertpapieren Liquidität zu beschaffen,

- $\quad$ Informationseffekte, so etwa die Wirkung der Verluste von Reserve Primary bei Lehman Brothers auf die Einschätzung der Anleger über die Position anderer Geldmarktfonds, die ähnliche Strategien verfolgt hatten.

Die Bundesregierung scheint vor allem Dominoeffekte aufgrund von Zahlungsausfällen im Auge zu haben, vielleicht auch die Furcht vor einer allgemeinen Panik entsprechend der im HREUntersuchungsausschuss genannten allgemeinen Panik nach dem Lehman-Konkurs. Es ist aber angebracht, die verschiedenen Ansteckungsmechanismen im Einzelnen zu prüfen.

Dominoeffekte aufgrund von Zahlungsausfällen oder aufgrund eines Fortfalls der Bank als potentiellem Vertragspartner setzen voraus, dass die betreffende Bank tatsächlich ausfällt. Im vorliegenden Zusammenhang ist diese Gefahr, wie oben ausgeführt, als sehr gering einzuschätzen, da erstens die in Rede stehenden Informationen über die betroffenen Banken nicht wirklich Neues enthielten und zweitens die Stützungsmaßnahmen des Bundes und der Länder geeignet waren, einen derartigen Ausfall zu verhindern.

43 Siehe M. Hellwig, Systemic Risk and Macro-Prudential Policy, in: A. Houben, R. Nijskens und M. Teunissen (eds.), Putting Macroprudential Policy to Work, Occasional Studies, Volume 12-7, De Nederlandsche Bank, Amsterdam, 2014, 42-77. 
Ansteckungseffekte aufgrund der Marktwirkungen der defensiven Maßnahmen von Banken spielten in den Krisen von 2008 und 2011 eine wichtige Rolle, das aber jeweils, weil die Banken konkrete Gründe für diese Maßnahmen hatten. Der Fortfall bzw. die Kontraktion der Geldmarktrefinanzierungen 2008 und 2011 veranlassten etliche Banken zu Wertpapierverkäufen, desgleichen die im Herbst 2011 vorgenommene Ankündigung einer Erhöhung der Eigenkapitalanforderungen. Im Kontext des November und Dezember 2010 sind entsprechende Gründe für ein durch Antworten der Bundesregierung auf die Fragen der Fraktion Bündnis 90/Die Grünen veranlasstes defensives Verhalten der Banken nicht ersichtlich.

Auch Ansteckungseffekte durch Informationszusammenhänge waren nicht zu erwarten. Wie im vorstehenden Teil ausgeführt und im genannten Fall der Geldmarktfonds nach dem Lehman-Konkurs bestätigt, orientiert sich das Anlegerverhalten auch in Krisen weitgehend an handfester institutionenspezifischer Information. Im gegebenen Kontext im Herbst 2010 war bekannt, welche Banken 2008 und 2009 Probleme gehabt hatten und welche Banken Stützungsmaßnahmen gebraucht hatten. Die Vorstellung, dass die Information über den Umgang der Aufsichtsbehörden mit einer dieser Banken aus Sicht der Anleger auch wesentliche neue Information über eine andere dieser Banken enthalten hätte - oder auch über ein Institut wie die Deutsche Bank oder eine Genossenschaftsbank, die keine staatliche Unterstützung in Anspruch genommen hatten, erscheint daher als abwegig.

\section{Zusammenfassung und Ergebnisse}

Die Entwicklungen, die zusammenfassend mit dem Wort „Finanzkrise“ bezeichnet werden, waren für den deutschen Steuerzahler insgesamt sehr teuer. Nach heutigem Stand ist von einer Belastung von mehr als € 70 Mrd. auszugehen. Jedoch hat es, anders als in den USA, in Deutschland keine umfassende Untersuchung der Krise(n) und der besonderen deutschen Betroffenheit gegeben. Die pauschale Aussage, man sei ein Opfer von Fehlentwicklungen in den USA geworden, hat von den maßgeblichen deutschen Beiträgen abgelenkt. Auch die seitherige Entwicklung ist von einem Mangel an öffentlicher Diskussion geprägt, was u.a. zur Folge hatte, dass Reformen wie das Bankenrestrukturierungsgesetz von 2010 nicht das leisten konnten, was versprochen wurde, und alsbald ergänzt werden mussten.

Die Antworten der Bundesregierung auf die Anfragen der Fraktion Bündnis 90/Die Grünen im November und Dezember 2010 passen in dieses Bild. Die für diese Antwort gegebenen Begründungen sind sachlich problematisch:

- Das Bild von „der Finanzkrise“ als einem fortdauernden Zustand, der eine Gefährdung von Finanzinstitutionen und Finanzsystem auch im Herbst 2010 begründete, ist sachlich falsch. Aus Sicht der Banken war 2010 überwiegend ein Jahr der Ruhe und der Stabilisierung. Die Turbulenzen des Folgejahrs wurden maßgeblich durch die Wendung der Politik im Umgang mit der griechischen Schuldenkrise begründet (Schuldenschnitt zu Lasten der privaten Gläubiger); offensichtlich hielt die Bundesregierung die Banken für stabil genug, die Folgen dieser Wendung auszuhalten.

- Wenn die Bundesregierung behauptet, bankspezifische Informationen über Sonderprüfungen der Aufsicht in den Jahren 2005-2008 könnten die Reputation und die Refinanzierungsmöglichkeiten der Banken gefährden, so übergeht sie die Tatsache, dass 2010 etliche dieser Banken gar nicht mehr selbständig im Markt waren und die anderen fast alle durch Kapitalbeiträge 
und Garantien des Bundes und/oder der Länder gestützt wurden. Dass diese Banken aufgrund ihrer früheren Tätigkeiten in Schwierigkeiten gekommen waren, war allseits bekannt.

- Die Aussage, in Ermangelung von Wortprotokollen könne man nichts über Wortmeldungen von Vertretern der Aufsicht und der Deutschen Bundesbank in Gremiensitzungen der Banken sagen, ist nicht glaubwürdig. Äußerungen der Behörden in solchen Sitzungen werden nicht ohne triftigen Grund getätigt. Man kann davon ausgehen, dass es über etwaige vorzubringende Anliegen behördeninterne Vorbereitungen und Abstimmungen gab. Wenn das im Nachhinein in der Öffentlichkeit oft beteuerte Unbehagen bezüglich der Liquiditätspolitik einzelner Institute oder der mit Zweckgesellschaften verbundenen Risiken in Gremiensitzungen der Banken geäußert wurde, so wird es darüber Unterlagen geben. Sollte es solche Unterlagen nicht geben, so liegt die Vermutung nahe, dass dieses Unbehagen nicht in Gremiensitzungen der Banken geäußert wurde.

- Die Vorstellung, es sei gefährlich, zusätzliche, auch scheinbar belanglose Informationen öffentlich zugänglich zu machen, da das eine unverhältnismäßige Reaktion des Finanzsystems hervorrufen könne, ist falsch. „Bank Runs“, Schalterstürme u.ä. kommen erfahrungsgemäß dort vor, wo es substantielle Gründe gibt, an der Solvenz der betreffenden Institution zu zweifeln. In einem solchen Fall ist es allerdings angebracht, die Probleme aktiv anzugehen und nicht durch Vertuschen auf die lange Bank zu schieben. Transparenz ist eine wichtige Grundlage für die sogenannte „dritte Säule“ des vom Basler Ausschuss für Bankenaufsicht erstellten Regelwerks.

- Auch das Risiko systemischer Ansteckungseffekte ist im gegebenen Kontext als gering zu veranschlagen. Von den bekannten Ansteckungseffekten im Finanzsystem kämen allenfalls Weiterungen von Refinanzierungsproblemen einer Bank infrage, z.B. weil diese Bank mit Zahlungen in Verzug kommt oder weil sie Notverkäufe von Anlagen tätigt und damit die Marktpreise drückt. Da die angesprochenen Banken alle vom Staat gestützt wurden, hatten sie keine Refinanzierungsprobleme, waren auch durch die Veröffentlichung der Information über die Vergangenheit keine neuen Refinanzierungsprobleme zu erwarten und dementsprechend auch keine Weiterungen solcher Probleme. 


\section{Anhang: Überblick über die im Verfahren zur Diskussion ste- henden Anfragen}

\subsection{Kleine Anfrage vom 11. November 2010 (BT-Drs. 17/3740 und BT-Drs. 17/4617)}

Frage 1: An welchen Aufsichtsrats- bzw. Verwaltungsratssitzungen oder sonstigen Sitzungen von Gremien mit Kontrollfunktionen haben jeweils Mitarbeiterinnen und Mitarbeiter der Deutschen Bundesbank bzw. der Bundesanstalt für Finanzdienstleistungsaufsicht (BaFin) in den Jahren 2005 bis 2008 teilgenommen, und in wie vielen davon haben sie das Wort ergriffen (mit der Bitte um institutionsspezifische Angaben jeweils für BaFin und Bundesbank und um institutsspezifische Angaben für BayernLB, Sachsen LB, West LB, HSH Nordbank, IKB Deutsche Industriebank, Düsseldorfer Hypothekenbank, Hypo Real Estate HRE, Commerzbank, Dresdner Bank)?

Hierauf antwortete die Bundesregierung, da keine Wortprotokolle geführt werden, könnten keine Angaben über Wortmeldungen gemacht werden. Im Übrigen könnten institutsspeczifische Angaben über Sitzungsteilnahmen nur in der Geheimschutzstelle des Deutschen Bundestages zur Verfügung gestellt werden.

Fragen 2 und 3: An wie vielen Aufsichtsratssitzungen u.ä. von Kreditinstituten haben Mitarbeiterinnen und Mitarbeiter der Deutschen Bundesbank bzw. der BaFin in den Jahren 2005 bis 2008 insgesamt teilgenommen? Wie viele derartige Sitzungen gab es?

Für die Bundesbank nannte die Antwort der Bundesregierung die erbetenen Zahlen für die angegebenen Jahre. Für die BaFin sei eine entsprechende Auskunft nur mit unzumutbarem Aufwand möglich, denn eine Statistik gebe es nicht und man müsse bei ca. 2000 Instituten mit teilweise mehreren Sitzungen pro Jahr die Unterlagen einzeln durchgehen. Wie viele Sitzungen es insgesamt gab, könne man nicht sagen, da die Institute nicht verpflichtet seien, die Aufsicht über die Anberaumung von Sitzungen zu informieren.

Fragen 4 und 5: Hier ging es um die Zahl der Aufsichtsgespräche in den Jahren 2005 - 2008, zum einen institutsspezifisch für die in Frage 1 genannten Institute, zum anderen insgesamt.

Die Antwort der Bundesregierung nennt institutsbezogene Zahlen für routinemäßige Aufsichtsgespräche. Dagegen wurden institutsspezifische Zahlen für anlassbezogene Aufsichtsgespräche nur in der Geheimschutzstelle des Deutschen Bundestags zur Verfügung gestellt. Für die Bundesbank wurden aggregierte Zahlen für routinemäßige und anlassbezogene Aufsichtsgespräche angegeben, für die BaFin wurden solche Zahlen nicht angegeben, mit derselben Begründung wie bei Frage 3 .

Fragen 6 und 7: Hier ging es um die Zahl der Sonderprüfungen in den Jahren 2005 - 2008, zum einen institutsspezifisch für die in Frage 1 genannten Institute, zum anderen insgesamt.

Die Antwort der Bundesregierung enthält nur die aggregierten Zahlen. Die institutsspezifischen Zahlen wurden nur in der Geheimschutzstelle des Bundestags zur Verfügung gestellt.

Frage 8: Diese Frage betraf den Umgang der Aufsicht mit Zweckgesellschaften wie Rhineland Funding (IKB) oder Ormond Quay (Sachsen LB). Im Einzelnen wurde gefragt nach etwaigen Gesprächen von Mitarbeitern der Aufsicht mit den betreffenden Instituten, nach der Wahrnehmung der Risiken 
insbesondere bei Landesbanken, bei denen durch die Gewährträgerhaftung auch der Steuerzahler im Risiko stand, schließlich nach den Maßnahmen, die solche Zweckgesellschaften nunmehr verhindern würden.

Auch hier wurden institutsspezifische Auskünfte nur in der Geheimschhutzstelle des Bundestags gegeben. Im allgemeinen führte die Bundesregierung aus, es habe etliche Gespräche und Korrespondenz gegeben, die BaFin sei regelmäßig davon ausgegangen, dass die Verwendung von Zweckgesellschaften und Conduits mit den aufsichtsrechtlichen Regeln vereinbar sei. Jedoch könne eine Umgehung aufsichtsrechtlicher Regeln nicht immer ausgeschlossen werden.

Frage 9: Diese Frage betraf eine Fehlmeldung der irischen HRE-Tochter Depfa in ihren Liquiditätsangaben Anfang August 2008 gegenüber den irischen Behörden.

Die Antwort der Bundesregierung verwies auf die Zuständigkeit der irischen Behörden, mit denen die deutschen Behörden allerdings im Informationsaustausch gestanden hätten.

Fragen 10, 12 und 13: Diese Fragen betrafen das Verhalten der Aufsichtsbehörden im Zusammenhang mit der Beteiligung der BayernLB an der österreichischen Hypo Group Alpe Adria.

Die Antwort der Bundesregierung verwies zum einen auf die unternehmerische Autonomie der Bank bei ihren Entscheidungen über eine derartige Akquisition, zum anderen auf das Vertraulichkeitsinteresse der Bank. Details wurden nur in der Geheimschutzstelle zur Verfügung gestellt.

Frage 11: Diese Frage betraf die Tätigkeit der Aufsicht im Umgang mit den internen Kontrollen und der Risikosteuerung bei HSH Nordbank (Omegageschäfte).

Die Bundesregierung verwies auf das Vertraulichkeitsinteresse der Bank und teilte Details nur in der Geheimschutzstelle mit.

Frage 14: Für die in Frage 1 genannten Institute wurde gefragt, in welche Felder der Zwölf-FelderMatrix der Risikoklassifizierung der BaFin die Institute in den Jahren 2005 bis 2008 jeweils eingestuft wurden.

Die Bundesregierung stellte diese Information nur in der Geheimschutzstelle des Bundestags zur Verfügung.

Frage 15: Diese Frage betraf tatsächliche und in Vorbereitung befindliche erhöhte Kapitalanforderungen nach $\S 10$ (1b) KWG und die Einstufung der Institute in der Zwölf-Felder-Matrix der Risikoklassifizierung der BaFin.

Die Bundesregierung nannte eine tatsächliche und zwölf mögliche derartige Anforderungen und gab die jeweiligen Risikoklassifizierungen an.

Frage 16: Hier wurde gefragt, ob unter den von WestLB in die Erste Abwicklungsanstalt eingebrachten Vermögenswerten auch solche waren, die von Goldman Sachs akquiriert worden waren.

Die Bundesregierung gab an, darüber keine Information zu haben. 
Frage 17: Diese Frage galt den von HSH Nordbank und von Hypo Real Estate in die Verbriefungstransaktion „St. Pancras“ eingebrachten Immobilienportfolios und die damit verbundenen Senkungen der Eigenkapitalanforderungen an die Banken.

Die Bundesregierung nannte für die Transaktion den Wert von jeweils rd. 3,45 Mrd. Euro und verwies für weitere Details auf die Geheimschutzstelle des Bundestags.

Frage 18: Hier wurde danach gefragt, in welchem Umfang Mitarbeiterinnen und Mitarbeiter von mit SoFFin-Mitteln unterstützten Banken unterhalb der Vorstandsebene Jahresgehälter in Höhe von mehr als $€ 500.000$ bezogen.

Die Bundesregierung betonte die Notwendigkeit der Vertraulichkeit und verwies die Fragesteller auf die Geheimschutzstelle des Bundestags.

\subsection{Schriftliche Anfragen vom 20. Dezember 2010 (BT-Drs. 17/4350)}

Nr. 34: Diese Frage betraf die Gründe für den Verkauf der IKB und den dabei erzielten Preis.

Eine öffentliche Auskunft über den Preis lehnte die Bundesregierung mit Verweis auf die vereinbarte Vertraulichkeit ab.

Nr. 35: Hier wurde nach Fällen gefragt, in denen die BaFin Banken, die in der Krise vom Bund gestützt worden waren, den Erwerb eigener Anleihen zu Preisen unter pari untersagt hatte.

Die Bundesregierung verwies auf das Gebot der Vertraulichkeit und verweigerte die Auskunft. 